\title{
Sharing Economy in der Elektrizitätswirtschaft: Treiber und Hemmnisse
}

\author{
Pia Szichta ${ }^{1,2}$ iD $\cdot$ Ingela Tietze ${ }^{3}$ iD \\ Eingegangen: 24. April 2020 / Überarbeitet: 17. Juli 2020 / Angenommen: 5. September 2020 / Online publiziert: 1. Oktober 2020 \\ (c) Der/die Autor(en) 2020
}

\section{Zusammenfassung}

Im Rahmen der Energiewende in Deutschland erfolgt eine schrittweise Dezentralisierung der Energieerzeugung und damit einhergehend nehmen Sharing-Angebote zu. Verglichen mit anderen Branchen ist das Sharing-Angebot in der Elektrizitätswirtschaft gering. In diesem Beitrag werden deshalb anhand einer Literaturrecherche und darauf basierenden Experteninterviews Treiber und Hemmnisse für Sharing-Angebote in der Elektrizitätswirtschaft identifiziert. Darüber hinaus werden begünstigende Rahmenbedingungen und Umweltwirkungen aus Expertensicht erhoben. In einer multiplen Fallanalyse werden aufbauend die Fallbeispiele Brooklyn Microgrid, Power Peers und die sonnenCommunity analysiert. Die Ergebnisse dieser Fallanalyse zeigen Heterogenitäten im Aufbau und in der praktischen Umsetzung, weshalb Verallgemeinerungen zu Treibern und Hemmnissen nur beschränkt möglich sind. Der in dem Beitrag beschriebene Systematisierungsansatz bietet allerdings die Möglichkeit, verschiedene Fallbeispiele inhaltlich differenziert zu untersuchen und miteinander zu vergleichen. Auf dieser Grundlage werden erste Handlungsempfehlungen für Anbieter und Politik abgeleitet, die eine Umsetzung von Sharing-Ansätzen in der Energiewirtschaft begünstigen.

Schlüsselwörter Sharing Economy $\cdot$ Energy Sharing $\cdot$ Platform Energy $\cdot$ Digital Energy $\cdot$ Peer-to-Peer Energy

\section{Title sharing economy in the electricity sector: drivers and barriers}

\begin{abstract}
Within the German Energy Transition, energy production is being gradually decentralised and, as a result, the offer of sharing services is increasing. However, compared to other sectors the increase of sharing services in the electricity sector is small. We therefore identify drivers and barriers for sharing services in the electricity sector using systematic literature reviews and expert interviews. Furthermore, environmental impacts and framework conditions for the Sharing Economy in the electricity sector were collected in the expert interviews. Using a multiple case analysis, the cases Brooklyn Microgrid, Power Peers and sonnenCommunity are analysed. The results of this case study show a heterogeneity in structure and practical implementation. Due to this heterogeneity, general drivers and barriers can only be derived to a limited extend. However, the developed systematic approach offers the opportunity to examine and compare different case studies in a differentiated way. On this basis, initial general recommendations for action for both providers and politics can be derived, that favour the implementation of sharing approaches in the energy industry.
\end{abstract}

Keywords Sharing Economy · Energy Sharing $\cdot$ Platform Energy $\cdot$ Digital Energy $\cdot$ Peer-to-Peer Energy

Pia Szichta

szichta@hfm.tum.de

1 TUM School of Life Sciences, Lehrstuhl für Holzwissenschaft, Technische Universität München (TUM), München, Deutschland

2 Hochschule Pforzheim, Pforzheim, Deutschland

3 Institut für Industrial Ecology (INEC), Hochschule Pforzheim, Pforzheim, Deutschland

\section{Einleitung}

Die Energiewende impliziert einen tiefgreifenden Wandel des Energiesektors. Die Elektrizitätswirtschaft betrifft dies in besonderem Maße, da mit dem Ausbau der erneuerbaren Energien eine Änderung der Besitzstrukturen verbunden ist: Während die meisten konventionellen Kraftwerke im Besitz von Energieversorgungsunternehmen sind, stel- 
len etwa $40 \%$ der erneuerbaren Anlagen Projekte unter Beteiligung privater Bürger dar (Einzelbesitz, Bürgerenergiegesellschaften und Bürgerbeteiligungen) (Heinrich-BöllStiftung et al. 2018). Durch die Beteiligung der lokalen Bevölkerung an erneuerbaren Energieprojekten wird das Gelingen der Energiewende unterstützt, da durch die Beteiligung Widerstände der Bevölkerung abgebaut werden können (Brinkmann und Schulz 2011; Poppen 2015).

Im Lauf der letzten Jahre ist die Zahl an dezentralen Erzeugungskapazitäten mit einer Verbindung zur Nachfrageseite der Stromsysteme stetig angestiegen (Zhou et al. 2018). Auf der Nachfrageseite treten zunehmend Prosumenten auf, die Strom sowohl bereitstellen als auch nachfragen (Li et al. 2019). Deren dezentrale Erzeugungskapazitäten können mithilfe von Onlineplattformen so vernetzt werden, dass Prosumenten in der Lage sind, über diese Plattformen Strom miteinander austauschen (Zhou et al. 2018).

Ansätze der Sharing Economy ermöglichen alternative Nutzungs- und Beteiligungsformen privater Bürger an Geschäftsprozessen. Bislang wurde kein Konsens über eine einheitliche Definition der Sharing Economy gefunden (Sundararajan 2016). Tietze (2020) identifiziert Gemeinsamkeiten verschiedener Definitionen der Sharing Economy bezüglich des Geteilten Guts, der Informationskanäle, dem Eigentum und Zugang sowie der Nutzung. Daher basiert diese Arbeit auf dem Verständnis der Sharing Economy in Form eines über ein Netzwerk sichergestelltes, zeitlich begrenztes Zugriffsrecht auf ein Produkt oder eine Dienstleistung mit dem Ziel der Erhöhung der Kapazitätsauslastung. Verbraucher können auf dieses Angebot der Ressourcenbesitzer beispielsweise über Peer-to-Peer-Plattformen zugreifen (Daunoriené et al. 2015). Die SharingModelle, bei denen Prosumenten oder Erzeuger mit Verbrauchern Strom, bzw. erneuerbare Energieerzeugungsanlagen und Speicherkapazitäten (Plewnia 2019) direkt und ohne einen konventionellen Energieversorger austauschen, werden auch als Peer-to-Peer (P2P) Energiehandel bezeichnet (Hamari et al. 2016).

Long et al. (2018) unterteilen bereits umgesetzte P2PSharing-Ansätze in der Energiewirtschaft in drei Kategorien:

- Blockchain-Lösungen: Diese Sharing-Modelle basieren auf der Durchführung und Verschlüsselung von Transaktionen mithilfe von Blockchain-Technologie.

- Online-Matching-Plattformen: Anbieter stellen eine Online-Plattform zur Verfügung, über die Teilnehmer ihren Stromverbrauch abgleichen können.

- Batteriespeicher-Communities: Der Einsatz von Batteriespeichern steht im Vordergrund des Sharing-Modells.

In diesem Zusammenhang ist allerdings anzumerken, dass der Energiesektor im Vergleich zu anderen Sektoren spezifische Charakteristika aufweist, die die Umsetzung von Sharing Economy erschweren (Tietze 2020). Durch den Einbezug finanzieller Transaktionen wird nicht der Strom selbst, sondern vielmehr die Kapazität erneuerbarer Energieerzeugungsanlagen wirtschaftlich geteilt. Darüber hinaus erfolgt aufgrund räumlicher Entfernung oftmals ein bilanzielles und kein physisches „Teilen“ des erzeugten Stroms. In Mikrogrids ist die räumliche Nähe allerdings gegeben und die finanziellen Transaktionen können dem physischen Stromfluss entsprechen (Plewnia 2019).

Im Transport- und Tourismussektor sind profitable und populäre Sharing-Geschäftsmodelle wie Uber, Couchsurfing und AirBnB entstanden, die mit einer höheren Flexibilität in den Sektoren einhergehen. Die Sharing Economy verspricht auch für den Energiesektor erhöhte Flexibilität (Zhou et al. 2018), welche aufgrund des Wandels zu höheren Anteilen erneuerbaren Energien im Rahmen der Energiewende erforderlich wird (Untersteller 2016). Inwieweit das Ziel einer umweltfreundlichen Energieversorgung durch Ansätze der Sharing Economy unterstützt wird, ist allerdings fraglich. Während einige Autoren Sharing Economy als grundsätzlich positiv für die Umwelt erachten, überwiegen aus Sicht anderer Autoren die negativen Auswirkungen auf die Umwelt (eine Übersicht der aktuellen Diskussionslage hierzu findet sich in Gossen et al. (2019)).

Vor dem Hintergrund der Potentiale der Sharing Economy zur Unterstützung der Transformation des Energiesystems durch Dezentralisierung und Flexibilisierung zielt dieser Beitrag auf die Identifikation von

- motivierenden Aspekten und Beweggründen für Nutzung, Umsetzung und Angebot (Treibern) und

- Aspekten mit hemmender oder erschwerender Auswirkung auf Nutzung, Umsetzung und Angebot (Hemmnissen)

von Sharing Economy in der Elektrizitätswirtschaft ab. Zur Erhebung von Treibern und Hemmnissen der Sharing Economy in der deutschen Elektrizitätswirtschaft wurden im Rahmen einer Masterthesis Experteninterviews durchgeführt. Zusätzlich werden die Erwartungen hinsichtlich der Umweltwirkungen von und Einschätzungen zu begünstigenden Rahmenbedingungen für Sharing Economy erfragt. Anhand von drei Fallbeispielen wird ergründet, inwieweit sich die zuvor identifizierten Treiber und Hemmnisse in der Praxis wiederfinden. Anschließend wird der Einfluss von begünstigenden Rahmenbedingungen zur Verstärkung der Treiber und dem Überwinden von Hemmnissen in den Fallbeispielen beschrieben. Zusätzlich werden die drei Fallbeispiele auf potenzielle Umweltwirkungen überprüft, bevor Implikationen im Hinblick auf eine Unterstützung der Transformation des Elektrizitätssystems abgeleitet werden. 


\section{Treiber und Hemmnisse von Ansätzen der Sharing Economy in der Literatur}

Dem von Booth (2006) beschriebenen Vorgehen zur systematischen Literaturrecherche folgend werden Treiber und Hemmnisse der Sharing Economy recherchiert. Die genutzten Plattformen sind Science Direct, Google Scholar, Springer Link, Scopus und Research Gate. Aus Aktualitätsgründen umfasst der Zeitrahmen die letzten fünf Jahre und die für die Recherche genutzten Schlagworte werden aus dem Themenfeld der Forschungsfrage abgeleitet: Blockchain, Blockchain Energy, Sharing Economy, Smart Grid, Platform Energy, Distributed Energy Resources, Peer to Peer, Energy Sharing, Self-Sufficiency, Micro Grid und Digital Energy (jeweils englisch und deutsch). Diese Liste wird durch die von Plewnia in seiner Arbeit zu Sharing-Geschäftsmodellen in der Energiewirtschaft verwendeten Begriffe ergänzt: Collaborative Economy, Cloud Energy, Demand Side Management und Peer Energy (Plewnia 2019). Im Folgenden werden die unter diesen Parametern in der Literatur identifizierten Treiber und Hemmnisse kurz zusammengefasst.

Finanzielle Vorteile stellen einen wichtigen Treiber für Sharing-Angebote dar:

- Der benötigte Strom kann aufgrund der Verringerung der Transaktionsebenen kostengünstig angeboten werden (Kloppenburg und Boekelo 2019; Pires Klein et al. 2020).

- Aufgrund der auslaufenden Förderung erneuerbarer Energien und der geringen Vergütung von Kraft-WärmeKopplungsanlagen werden alternative Geschäftsmodelle wie Sharing für viele Akteure attraktiv (Nicklaß et al. 2017). Sinkende Einspeisevergütungen für den Verkauf an das Stromnetz bieten Kunden in vielen Ländern finanzielle Vorteile, wenn sie Strom miteinander handeln oder tauschen, anstatt ihn direkt ins Netz einzuspeisen (Zhou et al. 2020).

- Eine Untersuchung von Celik et al. (2018) zeigt, dass eine effiziente Planung des Einsatzes der geteilten Güter mithilfe eines Algorithmus sowohl Kosten als auch die Spitzenlast in Sharing-Ansätzen reduzieren kann.

- Ein lokaler Speicher der, im Gegensatz zu einer Vielzahl individuell genutzter Speicher, von mehreren Haushalten gemeinsam genutzt wird, kann Kosten für die Energiespeicherung verringern (Parra et al. 2017). In Deutschland fehlen jedoch rechtliche Rahmenbedingungen für gemeinschaftlich genutzte Speicher (Gährs und Knoefel 2020).

Hackbarth und Löbbe (2020) verweisen allerdings auf das starke Interesse an innovativer Preisgestaltung in Form von Nutzungsdauertarifen oder inklusiven Leistungen, die
Interessenten einem allgemeinen finanziellen Vorteil vorziehen.

Ein weiterer Grund für das wachsende Interesse vieler Akteure an Sharing-Angeboten ist das zunehmende Umweltbewusstsein in der Bevölkerung: Der Nutzen für die Umwelt von erneuerbaren Erzeugungsanlagen wirkt sich vermehrt auf die Handlungen der Gesellschaft aus (Sun et al. 2018). Darüber hinaus können Interessenten ein Sharing-Angebot in Anspruch nehmen und ihren Strom aus erneuerbarer Energieerzeugung beziehen, selbst wenn sie nicht über genügend Eigenkapital verfügen, um in eine eigene Solaranlage oder einen Batteriespeicher zu investieren (Tang et al. 2019). Allerdings können durch die Installation und Nutzung erneuerbarer Energieerzeugungsanlagen unabhängig von einem Sharing-Angebot positive Umweltwirkungen erzielt werden.

Anfangsinvestitionen stellen demgegenüber ein Hemmnis für die Inanspruchnahme von Sharing-Angeboten durch Privatpersonen dar (Kröhling 2017). Die Erfassung und Übermittlung von Daten und Informationen im Smart-Grid gelten für einen Teil der Kunden als weiteres Hemmnis (Peer et al. 2014), da sich eine wachsende Unsicherheit in Bezug auf eine mögliche Verletzung der Privatsphäre feststellen lässt (Otuoze et al. 2018). Die Befürchtung von Stromausfällen aufgrund von Sicherheitsbedrohungen wie Cyberhacking der Sharing-Systeme sind in diesem Zusammenhang ebenfalls ein Hindernis (Otuoze et al. 2018). Pasimeni (2019) verweist diesbezüglich allerdings auf eine höhere Versorgungssicherheit durch dezentralisierte Systeme wie Mikrogrids. Energiespeichersysteme erhöhen deren Stabilität zusätzlich (Han et al. 2020), jedoch gehen diese mit höheren Investitionskosten einher (Hoffmann et al. 2018).

Ein Hemmnis aus Anbietersicht ist das Fehlen einer allgemein akzeptierten interoperablen Schnittstelle. Eine solche Schnittstelle in Form einer Informationsplattform soll den effizienten Austausch von Informationen ermöglichen und für alle beteiligten Akteure leicht zugänglich sein. Dabei bewerkstelligt sie den Zugang der Beteiligten zu wichtigen Informationen und sichert gleichzeitig die Erfüllung der Geschäftsanforderungen (Tikka et al. 2019).

Darüber hinaus stellt die Komplexität und Intransparenz der rechtlichen Regulatorien ein Hemmnis für die Umsetzung von Sharing-Ansätzen dar. Die zusätzliche finanzielle Belastung, die mit der Nutzung eines Batteriespeichers im öffentlichen Netz einhergeht, gilt im Zusammenhang mit rechtlichen Rahmenbedingungen als wichtiges Hemmnis. Überdies können geschlossene Verteilnetze, die gemeinschaftlich genutzte, lokale Energiespeicherung vorantreiben würden, bislang ausschließlich von gewerblichen und industriellen Kunden genutzt werden (Gährs und Knoefel 2020). Des Weiteren wird in Bezug auf Speicherlösungen auf Bedenken hinsichtlich der Lebensdauer und 
Garantie sowie technischen Risiken aufmerksam gemacht (Hoffmann et al. 2018). Demnach spielt das Einhalten des Leistungsversprechens aus Nutzersicht offenbar eine wichtige Rolle.

Die identifizierten Treiber beschränken sich auf die drei Hauptaspekte Kostenvorteile, Umweltbewusstsein und Möglichkeit zur Partizipation im Rahmen der Teilnahme an Sharing- Modellen in der Elektrizitätswirtschaft. Das Spektrum der Hemmnisse ist breiter gefächert und deckt dabei sowohl die Angebots- als auch Nachfrageseite ab. Auf den ersten Blick konträr wirken der Treiber Partizipation, da keine eigenen Anlagen benötigt werden, und das Hemmnis der Anfangsinvestition für Privatpersonen. Dieser Widerspruch zeigt, dass nicht alle Treiber und Hemmnisse allgemeingültig für die Sharing Economy in der Elektrizitätswirtschaft sind, sondern eine Abhängigkeit von der Ausgestaltung des Sharing-Angebots besteht.

\section{Methoden}

Zur Erhebung von Treibern und Hemmnissen der Sharing Economy in der deutschen Elektrizitätswirtschaft werden Experteninterviews durchgeführt und anhand einer multiplen Fallanalyse Erkenntnisse zum Auftreten von Treibern und Hemmnissen in der Praxis gewonnen.

\subsection{Experteninterviews}

Experteninterviews werden durchgeführt, um auf das Wissen, die Handlungsorientierungen und -empfehlungen sowie Einschätzungen von Experten zugreifen zu können (Bogner et al. 2014). Die Experteninterviews im Rahmen der vorliegenden Arbeit fokussieren sich auf

- Deutungswissen, das die subjektive Perspektive der Experten, die Sichtweisen, Interpretationen sowie Sinnentwürfe enthält, und

- Prozesswissen, das auf Erfahrungen basiert, die im Rahmen einer persönlichen Nähe zum untersuchten Themenbereich gemacht wurden (Bogner et al. 2014).

Die im Vorfeld durchgeführte Literaturrecherche dient sowohl dazu, die Themenbereiche des Experteninterviews inhaltlich vorzubereiten als auch die Leitfaden-Fragen zu präzisieren. Bei der Konzeption des Leitfadens der vorliegenden Untersuchung wird nach Helfferich (2019) vorgegangen. Diese Vorgehensweise ermöglicht ein teilnarratives Interview, das aus einem ausgewogenen Verhältnis an Erzählaufforderungen und nachfolgenden Fragen besteht. Aufgrund der Heterogenität der Experten kann mit abweichenden Interviewschwerpunkten gerechnet werden. Dieses Vorgehen ermöglicht einerseits Flexibilität im Interview, und stellt andererseits die Vollständigkeit und Vergleichbar-
Tab. 1 Interviewleitfaden mit Hauptfragen und Teilaspekten

Hauptfrage Treiber: Welche Anreize bestehen für Privatpersonen oder Unternehmen, Sharing-Angebote zu nutzen?

- Welche Anreize werden seitens der Gesetzgebung geschaffen, Sharing-Angebote zu nutzen?

- Von welchen Vorteilen profitieren Privatpersonen oder Unternehmen, indem sie ihren Strom über Sharing-Angebote beziehen?

- Inwieweit trägt ein Umdenken in der Gesellschaft zu einer erhöhten Nutzungsbereitschaft von Sharing-Angeboten bei?

Hauptfrage Hemmnisse: Wo sehen Sie Hemmnisse in der Umsetzung/Weiterentwicklung/Verbreitung von Sharing-Angeboten

- Was hindert Unternehmen oder Privatpersonen daran, Sharing-Angebote in Anspruch zu nehmen?

- Mit welchen Bedenken gegenüber Sharing-Angeboten sind Sie persönlich schon in Berührung gekommen?

- An welchen Stellen gibt es Hindernisse in der praktischen Umsetzung von Sharing-Angeboten?

- Sehen Sie verschiedene Hinderungsgründe oder werden immer wieder dieselben Bedenken genannt?

- Sehen Sie Hindernisse im Bereich Datenschutz?

Hauptfrage Umweltwirkungen: Welche Auswirkungen auf die Umwelt hat eine Sharing-Economy Ihrer Ansicht nach?

- Welche Handlungen von Nutzern von Sharing-Angeboten wirken sich möglicherweise positiv auf die Umwelt aus?

- Welche Handlungen von Privatpersonen oder Unternehmen innerhalb der Sharing-Angebote wirken sich möglicherweise negativ auf die Umwelt aus?

- Welche Rebound- (Rückkoppelungs-) Risiken sehen Sie in Bezug auf die Sharing-Economy?

Hauptfrage Rahmenbedingungen: Welche Rahmenbedingungen sollten geschaffen werden, um die Bedenken zu berücksichtigen und die Treiber zu verstärken?

- Wie kann die Wirkung der bereits genannten Treiber verstärkt werden?

- Auf welchem Weg können die genannten Hindernisse für Sharing-Ansätze überwunden werden?

- Welche Anreize sind von politischer Seite aus notwendig, um eine Verbreitung der Sharing-Möglichkeiten zu gewährleisten?

- Wie sollten die Sharing-Angebote gestaltet werden, um negative Umweltfolgen zu verringern oder zu verhindern?

- Welchen Handlungsbedarf sehen Sie seitens der Wirtschaft?

- Wie müsste der Elektrizitätssektor der Zukunft aussehen, damit mehr Menschen Sharing-Angebote nutzen?

keit der Interviews sicher. Der Leitfaden mit Hauptfragen und Teilaspekten ist aus Tab. 1 ersichtlich.

Bei der Expertenauswahl wird auf eine Heterogenität der Stichprobe geachtet: Einerseits soll ein Praxisbezug auf verschiedenen Ebenen hergestellt und andererseits verschiedene Sichtweisen berücksichtigt werden. Das ermöglicht Lücken zu schließen, die innerhalb der Literaturrecherche entstanden sein können (Bogner et al. 2014). Relevante Akteure im Bereich der Energiewirtschaft, insbesondere in Bezug auf die Energiewende in Deutschland, sind politische Entscheidungsträger und Institutionen, Energieversorger, Privatpersonen, Energiegenossenschaften und dezentrale Initiativen in Kommunen und Regionen (Ohlhorst 2018). 
Tab. 2 Für Experteninterviews ausgewählte Stichprobe

\begin{tabular}{ll}
\hline Relevante Akteure im Bereich der Energiewirtschaft & Tätigkeitsbereich der ausgewählten Experten \\
\hline Politische Entscheidungsträger und Institutionen & Experte 1: Amt für Umweltschutz Stuttgart, Bereich Energiewirtschaft \\
& Experte 2: Bundestagspartei, Bereich Energiewirtschaft \\
Energieversorger & Experte 3: Energieversorger, Bereich Innovation \\
& Experte 4: Energieversorger, Bereich Produktmanagement Photovoltaikanlagen \\
Energiegenossenschaften & Experte 5: Genossenschaftsverband, Bereich Betreuung von Energiegenossenschaften \\
Dezentrale Initiativen & Experte 6: Städtischer Verein, Bereich erneuerbare Energien (Photovoltaik) \\
\hline
\end{tabular}

Tab. 3 Deduktiver Kodierleitfaden

\begin{tabular}{|c|c|c|c|}
\hline Kategorie & $\begin{array}{l}\text { Definition in Anlehnung an die Be- } \\
\text { griffsdefinition im Duden }\end{array}$ & $\begin{array}{l}\text { Ankerbeispiele aus Experteninter- } \\
\text { views }\end{array}$ & Kodierregel \\
\hline Treiber & $\begin{array}{l}\text { Ein Aspekt, der motivierend wirkt/ } \\
\text { einen Beweggrund darstellt, Sharing- } \\
\text { Angebote zu nutzen, umzusetzen oder } \\
\text { anzubieten }\end{array}$ & $\begin{array}{l}\text { Dann gibt es noch den Teil der Be- } \\
\text { völkerung, der etwas Gutes für die } \\
\text { Umwelt tun möchte und bemüht ist, } \\
\text { etwas aktiv voranzutreiben }\end{array}$ & $\begin{array}{l}\text { Prägnanter Grund, aus dem Personen oder } \\
\text { Unternehmen Sharing-Modelle in Anspruch } \\
\text { nehmen oder anbieten }\end{array}$ \\
\hline Hemmnisse & $\begin{array}{l}\text { Ein Aspekt der sich hemmend bzw. } \\
\text { erschwerend auf die Nutzung, Umset- } \\
\text { zung oder das Angebot von Sharing- } \\
\text { Angeboten auswirkt/ein Hindernis } \\
\text { darstellt }\end{array}$ & $\begin{array}{l}\text { Ein Thema ist immer die Verände- } \\
\text { rung. Die Interessenten denken, wenn } \\
\text { Sie etwas auf ihr Dach bauen, diese } \\
\text { Veränderung nachteilig ist }\end{array}$ & $\begin{array}{l}\text { Aspekt, der Personen oder Unternehmen } \\
\text { davon abhält, an Sharing-Modellen teilzu- } \\
\text { nehmen oder diese anzubieten }\end{array}$ \\
\hline $\begin{array}{l}\text { Umwelt- } \\
\text { wirkungen }\end{array}$ & $\begin{array}{l}\text { Eine durch ein Sharing-Modell verur- } \\
\text { sachte Veränderung bzw. Beeinflus- } \\
\text { sung der Umwelt }\end{array}$ & $\begin{array}{l}\text { Der Anschluss erneuerbarer Stromer- } \\
\text { zeugungsanlagen bedeutet einen } \\
\text { größeren Teil an Ökostrom im Netz, } \\
\text { der sich in Sachen } \mathrm{CO}_{2} \text { positiv auf die } \\
\text { Umwelt auswirkt }\end{array}$ & $\begin{array}{l}\text { Auf Sharing-Modellen basierende nachvoll- } \\
\text { ziehbare Handlungen oder Gegebenheiten, } \\
\text { die positive oder negative Umweltwirkun- } \\
\text { gen implizieren, selbst wenn sie nicht präzi- } \\
\text { se beschrieben werden }\end{array}$ \\
\hline $\begin{array}{l}\text { Begünsti- } \\
\text { gende } \\
\text { Rahmen- } \\
\text { bedin- } \\
\text { gungen }\end{array}$ & $\begin{array}{l}\text { Eine für Sharing-Modelle begünsti- } \\
\text { gende Bedingung, die gezielt dafür } \\
\text { geschaffen wird, den äußeren Rahmen } \\
\text { für das Angebot, die Umsetzung und } \\
\text { Nutzung abzustecken }\end{array}$ & $\begin{array}{l}\text { Die Lieferung im örtlichen Zusam- } \\
\text { menhang sollte gesetzlich klarer } \\
\text { behandelt und Abgaben minimiert } \\
\text { werden }\end{array}$ & $\begin{array}{l}\text { Konkrete Voraussetzungen bzw. } \\
\text { Verbesserungs-/Änderungsvorschläge, die } \\
\text { eine gesteigerte Nutzung oder Verringerung } \\
\text { negativer Umweltfolgen erzielen }\end{array}$ \\
\hline
\end{tabular}

Die Wahl der Experten fällt aus diesen Gründen auf Personen innerhalb Deutschlands, die sowohl einen Einblick in die Anbieterseite als auch in die Nutzerseite haben. Diese Einblicke werden zum einen durch ihr berufliches Umfeld und zum anderen durch den Kontakt zu Privatpersonen gewährleistet. Vor diesem Hintergrund wurde die aus Tab. 2 ersichtliche Stichprobe befragt.

Die Experteninterviews werden anhand der qualitativen Inhaltsanalyse nach Mayring und Brunner (2009) ausgewertet, die besonders für Transkripte von halb-strukturierten Interviews geeignet ist. Einzelne Interviewpassagen werden hierbei vorab bestimmten Kategorien zugeordnet. Im Rahmen einer deduktiven Kategorienbildung werden die vier Dimensionen Treiber, Hemmnisse, Umweltwirkungen und Rahmenbedingungen als Kategorien übernommen. Die Zuordnung der Interviewpassagen zu den Kategorien wird durch den in Tab. 3 dargestellten Kodierleitfaden, der eine präzise Definition der Kategorien, Kodierregeln und Ankerbeispiele enthält, unterstützt. Darauf aufbauend wird eine qualitative Analyse des Interviewmaterials durchgeführt. Innerhalb der zuvor gebildeten Kategorien werden Gemein- samkeiten, Unterschiede sowie Widersprüche herausgearbeitet und zusammengefasst.

\subsection{Multiple Fallanalyse}

Mit einer multiplen Fallanalyse wird überprüft, inwieweit sich die Einschätzungen zu Treibern und Hemmnissen aus den Interviews in der Praxis wiederfinden lassen. Darüber hinaus finden die Umweltwirkungen der Fallbeispiele besondere Beachtung, um einen Beitrag zur aktuellen Kontroverse zu den ökologischen Auswirkungen der Sharing Economy zu leisten.

Das Vorgehen im Rahmen der multiplen Fallanalyse orientiert sich an Yin (2018). Die übergreifenden Schritte stellen dabei (a) die Definition und das Design (b) das Vorbereiten, Sammeln und Analysieren sowie (c) das Analysieren und Schlussfolgern dar. Die zu überprüfende Theorie basiert auf der im Vorfeld durchgeführten Literaturrecherche und den Ergebnissen aus den Experteninterviews (a):

1. Innerhalb der Sharing Economy finden sich in Abhängigkeit der Umsetzungsformen zum einen gemeinsame 
und zum anderen unterschiedliche Treiber wie Hemmnisse wieder.

2. Es existieren gemeinsame und unterschiedliche Treiber wie Hemmnisse, die sich auf die Umweltwirkungen der Sharing-Ansätze auswirken.

Auf Basis der gezielten Auswahl dreier Fälle werden Einzelfallbeispiele individuell analysiert (b). In einer anschließenden Vergleichsanalyse (Hoffmann 2016) werden diese Einzelfallbeispiele einander gegenübergestellt, Zusammenhänge analysiert und fallübergreifende Schlussfolgerungen bezüglich des Untersuchungsgegenstands gezogen (c) (Yin 2018).

Eine solche Fallanalyse weist üblicherweise nicht die detaillierte Tiefe einer Einzelfallanalyse auf, sondern hebt auf Unterschiede und Vielfalt der verschiedenen Beispiele ab (Hoffmann 2016). Mithilfe der Untersuchung verschiedener Fallbeispiele ist es möglich, Unterschiede aufzudecken, verschiedene Fälle voneinander abzugrenzen oder Gemeinsamkeiten festzustellen. Die Fallbeispiele sollen einerseits größtmögliche Vielfalt und anderseits eine ausreichende Schnittmenge an Gemeinsamkeiten für einen Vergleich aufweisen (Eisenhardt und Graebner 2007). Um eine möglichst große Bandbreite der praktischen Umsetzungsformen in der Sharing-Economy in der Elektrizitätswirtschaft abzudecken, wird deshalb anhand der Systematisierung der P2P-Sharing-Ansätze jeweils ein Fall aus den drei Kategorien analysiert:

- Brooklyn Microgrid 101 als Vertreter der BlockchainLösungen,

- Powerpeers als Vertreter der Online-Matching-Plattformen und

- sonnenCommunity als Vertreter der BatteriespeicherCommunities.

Die für die Interviews ausgewählten Experten kommen ausschließlich aus Deutschland. Daher befinden sich zur Erweiterung der räumlichen Perspektive die Fallbeispiele neben Deutschland in den Niederlanden und den USA. Die Fallbeispiele unterliegen demnach verschiedenen gesetzlichen Voraussetzungen, die sich auf die Gestaltung der Sharing-Angebote auswirken können. Die gesetzlichen Rahmenbedingungen werden in dieser Arbeit allerdings nicht im Detail verglichen, da hierzu eine vertiefte Auseinandersetzung mit der Gesetzgebung in den einzelnen Ländern erforderlich ist. Es werden allerdings zentrale Spezifika hervorgehoben.

Die multiple Fallanalyse wird zur Sicherstellung eines systematischen und nachvollziehbaren Vergleichs anhand von Kriterien durchgeführt. Damit unterliegen die verschiedenen Fälle einem identischen Untersuchungsdesign und die Vorgehensweise wird in den jeweiligen Einzelfallanalysen repliziert (Yin 2018). Die für die Fallanalysen verwen- deten Kriterien stützen sich im Wesentlichen auf die Arbeiten von Plewnia (2019), Plewnia und Guenther (2018) und Bocken et al. (2014). Durch eine Erweiterung der Kategorie „Sektorenzugehörigkeit“ zu „Allgemeine Informationen" resultieren die folgenden vier Kriterienkategorien für die Systematisierung der Fallbeispiele: (1) Allgemeine Informationen, (2) Geteiltes Gut, (3) Marktorientierung und (4) Marktstruktur.

In die Kategorie (1) Allgemeine Informationen fallen zusätzlich zum Industriesektor nach Zhang et al. (2017) die Kriterien Projektname, zugehöriges Land, offizieller Startzeitpunkt des Projektes und das Ziel des Fallbeispiels. Das Ziel beinhaltet die Schlüsselaktivitäten sowie die Funktionsweise und somit eine Kurzbeschreibung des Hauptnutzens des Fallbeispiels nach Bocken et al. (2014).

Die zweite Kriterienkategorie umfasst die Kriterien zum (2) Geteilten Gut nach Plewnia (2019) mit Ergänzungen nach Bocken et al. (2014):

- geteiltes Material/geteilte Energie,

- Produktservicesystem: kurzfristige Vermietung oder ergebnisbasiertes Produktservicesystem,

- Raum, Ressourcen und Technologien,

- Daten und Informationen,

- Wissen und Weiterbildung,

- Erweiterbarkeit und

- Lebensdauer.

Die Kriterien zur (3) Marktorientierung dienen zur Einordnung der ökonomischen Orientierung der Fallbeispiele. Das Kriterium Ertragsmodell beschreibt in Anlehnung an Bocken et al. (2014) die Art und Weise der Erwirtschaftung des Einkommens innerhalb der Fallbeispiele. Zusätzlich wird entsprechend (Plewnia 2019) unterschieden, ob es sich um eine Online- oder Offlineplattform handelt. Die Netzwerkgröße gemäß Zhang et al. (2017) wird zur Beschreibung der Weitläufigkeit der jeweiligen Netzwerke herangezogen.

Die Kategorie (4) Marktstruktur bezieht sich auf den Aufbau der Fallbeispiele bezüglich ihrer Betreiber, deren Partner und ihrem Kundensegment nach Bocken et al. (2014), Plewnia (2019) und Zhang et al. (2017).

Zur Untersuchung der Umweltwirkungen der Fallbeispiele werden Kriterien nachhaltiger Geschäftsmodelle herangezogen. Bocken et al. (2014) führen Archetypen für nachhaltige Geschäftsmodelle ein, die sich anhand ihrer Hauptinnovation unterscheiden. Die Hauptinnovationen sind dreigeteilt in technologische, soziale und organisatorische Innovationen. Diese Aufteilung dient dazu, spezifische Lösungen zu finden, die die jeweiligen Geschäftsmodelle in Richtung Nachhaltigkeit bewegen können. Die Sharing Economy lässt sich dem Archetypen „Bereitstellung von Funktionalität anstatt Besitz“ zuordnen. Darüber hinaus sind die Archetypen „Substituieren mit erneuerbaren 
Tab. 4 Kriterienkatalog zur Analyse der Umweltwirkungen

Merkmale

Konsumentenbildung, reduzierter Ressourcenverbrauch

Maximierung des Verbrauchernutzens

Verringerung des Produktionsdurchsatzes

Langlebigkeit, Haltbarkeit, Lieferantenauswahl nach Haltbarkeit

Erweiterbarkeit

Verzicht auf nicht erneuerbare Ressourcen, Verringerung des Einsatzes nicht erneuerbarer Quellen

Reparaturfähigkeit

Keine Rabatte, kein Overselling

Wieder- und Weiterverwendbarkeit

Verringerung von Emissionen

Verringerung der Deponierung synthetischer Abfälle

Energien“ und „Förderung von Suffizienz“ relevant für Sharing-Ansätze in der Elektrizitätswirtschaft. Die aus den Archetypen abgeleiteten Kriterien finden sich in Tab. 4. Zur Analyse der Umweltwirkungen werden diese um die Ergebnisse aus den Experteninterviews ergänzt.

\section{Ergebnisse}

Nachstehend werden zuerst die Erkenntnisse aus den Experteninterviews dargestellt. Von den Experten angeführte Treiber und Hemmnisse werden mit denen der Literatur zusammengeführt und zur Analyse der Fallbeispiele herangezogen. Entsprechend wird hinsichtlich der von den Experten erwarteten Umweltwirkungen der Sharing-Ansätze verfahren. Zudem wird geprüft, inwieweit sich die von den Experten als begünstigend eingeschätzten Rahmenbedingungen auf die Fallbeispiele auswirken.

\subsection{Erkenntnisse aus den Experteninterviews}

Aus den von den verschiedenen Experten genannten Treibern lassen sich fünf zentrale Themenbereiche ableiten, die jeweils von mindestens vier der Experten genannt werden:

- Möglichkeit zu Partizipation: Diesen Treiber führen fünf der sechs befragten Experten an. Sowohl kleine Unternehmen als auch Privatpersonen erhalten mithilfe von Sharing-Modellen die Möglichkeit, Strom direkt aus erneuerbarer Energieerzeugung zu beziehen. Ohne selbst Flächen für erneuerbare Erzeugungsanlagen zu besitzen, können sie auf diese Weise den Strommarkt mitgestalten und v. a. Strom aus ihrer eigenen Umgebung beziehen. Diesbezüglich spielt die Regionalität eine entscheidende Rolle. Bestimmte Sharing-Angebote ermöglichen den Nutzern einen Bezug von Strom aus erneuerbaren Energieerzeugungsanlagen in der eigenen Umgebung, sowie die Möglichkeit selbst zu bestimmen, woher der benötigte Strom kommt.

- Erneuerbare-Energien-Gesetz (EEG): Einen zentralen Treiber für die Inanspruchnahme von Sharing-Angeboten sehen vier Experten in der Unsicherheit von Anlagenbesitzern nach Ablauf der EEG-Förderung. Es wächst Interesse an alternativen Lösungsansätzen wie Sharing, da solche Ansätze eine Perspektive bieten, den Strom auch in Zukunft sinnvoll nutzen zu können.

- Abkehr von traditionellem Versorger: Vier Experten zufolge sehen Privatpersonen und Unternehmen in SharingModellen die Möglichkeit, sich von Energieversorgern zu lösen und dadurch Anhängigkeiten zu minimieren. Durch Eigenversorgung und das Teilen mit weiteren Mitgliedern von Sharing-Modellen wird ihnen Autarkie ermöglicht.

- Kostenvorteile: Diese werden von vier Experten als Treiber für Privatpersonen und Unternehmen angeführt, Sharing-Modelle nachzufragen als auch anzubieten.

- Unterstützung Umweltbewusstsein: Ein Umdenken der Bevölkerung in Bezug auf die Umwelt fungiert laut vier Experten als Treiber. Mithilfe von Sharing-Modellen kann die Energiewende aktiv durch Einzelne vorangetrieben werden.

Die von den Experten genannten Hemmnisse lassen sich ebenfalls fünf Themenbereichen zuordnen: Die Aussagen der Experten betreffen Aufwand, Wirtschaftlichkeit, Widerstand Netzbetreiber, Umsetzung und Einstellung:

- Aufwand: Allgemein wird hoher Aufwand von vier Experten als Hemmnis für Sharing-Modelle genannt. Als Unteraspekte werden Sicherstellen der Grundversorgung und Rechtskonformität, Verwaltungsaufwand und die Plattformkonzeption angeführt.

- Wirtschaftlichkeit: Mit dem hohen Aufwand einhergehende Kosten sehen vier Experten ebenfalls als Hemmnis. Dazu zählen auf der Nutzerseite hohe Anfangsinvestitionen, zu lange Amortisationsdauern, zu hohe Steuern, Netzentgelte und Umlagen sowie zu teure Speicherlösungen. Auf der Angebotsseite werden die individuelle Abwicklung bzw. Abrechnung, der Leitungsbau oder der Aufbau einer Plattform als zu kostenintensiv erachtet.

- Widerstand Netzbetreiber: Netzbetreiber sprechen sich möglicherweise gegen Sharing-Angebote aus, da die hohen Spannungsebenen nicht genutzt werden müssen.

- Einstellung: Die grundsätzliche Einstellung wird von vier Experten als Treiber angeführt, jedoch sehen drei der Experten darin ebenfalls ein Hemmnis: Bedenken hinsichtlich der Einhaltung des Leistungsversprechens und der Offenlegung von Daten wirken hemmend. 
- Umsetzung: Schlechte Erfahrungen mit der praktischen Umsetzung der ersten Sharing-Angebote stellen ein weiteres Hemmnis dar.

Die innerhalb der Experteninterviews von mindestens vier Experten thematisierten begünstigenden Rahmenbedingungen zur Verstärkung der Treiber bzw. zum Verringern der Hemmnisse lassen sich den vier Hauptthemenfeldern gesetzliche Anreize, Prozesseinfachheit, Impulse und Infrastruktur zuordnen:

- Das Thema gesetzliche Anreize wird von jedem der interviewten Experten angesprochen. Fünf Experten erachten eine $\mathrm{CO}_{2}$-Besteuerung als hilfreich für Sharing-Angebote, während das EEG in seiner jetzigen Form kritisch hinterfragt wird. Zwei Experten sprechen sich gegen eigene Anreizsysteme für Sharing-Modelle aus und fordern stattdessen ein gerechtes Gesamtsystem und Unterstützung von Sharing-Modellen durch Musterverträge und Normungsarbeiten.

- Eine Vereinfachung der bestehenden Prozesse und ein Abbau von bürokratischen Hindernissen sollten laut allen Experten erfolgen. Konkrete Vorschläge hierzu betreffen die Standardisierung von Formularen und Verträgen, Vereinfachung von Anträgen für Erzeugungsanlagen, eine transparente Gestaltung der Netzleistungskosten in Bezug auf Eigenstromnutzung und Steuerzahlung sowie eine Vereinfachung in der Lieferung auf lokaler Ebene.

- Die Verbesserung der Infrastruktur wird von allen Experten als Notwendigkeit für ein Fortschreiten der Sharing-Angebote angesprochen. Beispielsweise werden jederzeit verfügbares Internet, lokale Speicher, bidirektionaler Stromfluss und Plattformen als Voraussetzung erachtet.

- Anregungen von unterschiedlichster Seite werden von vier Experten als Verstärkung der Treiber erachtet: Regierungsebene, Bildungssystem und Unternehmen werden als relevante Impulsgeber genannt.

Die zentralen Aussagen der Experten zu positiven Umweltwirkungen der Sharing Economy in der Elektrizitätswirtschaft lassen sich auf die Themenfelder Nachfragereduktion und Anteil an erneuerbaren Energien reduzieren:

- Fünf Experten erwarten positive Umweltauswirkungen durch Sharing Economy aufgrund der Zunahme des Anteils an erneuerbaren Energieerzeugungsanlagen. So sind $\mathrm{CO}_{2}$-Einsparungen auf den steigenden Anteil der Menge an Strom, die aufgrund von Sharing-Modellen auf umweltfreundlicher Energieerzeugung basiert, zurückzuführen.

- Vier Experten sehen eine positive Auswirkung auf die Umwelt durch Einsparungen, die Sharing-Modelle hervorrufen. Durch die Inanspruchnahme von Sharing-An- geboten steigen das Interesse und das Bewusstsein für den eigenen Stromverbrauch an, was die Bereitschaft zur Reduktion des Stromverbrauchs erhöht. Die positive Umweltwirkung durch die Senkung des Stromverbrauchs wird von den Experten v. a. in einer Reduktion der $\mathrm{CO}_{2-}$ Emissionen gesehen.

Der Aspekt Ressourcennutzung wird von einem Experten kritisch hinterfragt: Einerseits kann der Ressourcenbedarf durch die gemeinsame Nutzung von Anlagen verringert werden. Andererseits bestehen einige Sharing-Angebote auf den Aufbau von dezentralen Speicherlösungen bei jedem Anwender, ohne zu hinterfragen, inwieweit übergreifende Speicherlösungen für den Ressourcenverbrauch vorteilhafter sind. Hinsichtlich des Auftretens von Reboundeffekten sind die Experten unterschiedlicher Meinung: Ein Experte stellt fest, dass Kunden sich weder für ihren Verbrauch noch für ihre Stromrechnung interessieren und bspw. Flatrates daher nicht zu einem gesteigerten Stromverbrauch führen würden. Demgegenüber sehen zwei Experten Reboundeffekte als Risiko von Sharing-Modellen: Der Bezug von Strom aus erneuerbaren Energiequellen könne Nutzer dazu verleiten, mehr Strom zu verbrauchen als zuvor.

Insgesamt zeigen sich innerhalb der Kategorien Treiber und Hemmnisse viele Gemeinsamkeiten in den Ausführungen. Die Experten beleuchten die Sharing-Modelle dabei von verschiedenen Seiten und Standpunkten, ohne sich inhaltlich zu widersprechen. Die in der Literatur genannten Treiber finanzielle Vorteile, Umweltbewusstsein und Möglichkeit zur Partizipation werden von den Experten ebenfalls angeführt, zusätzlich sehen die Experten einen Treiber in der Möglichkeit zur Abkehr von traditionellen Energieversorgern. Ebenso werden die Hemmnisse aus der Literatur von den Experten bestätigt: Die Höhe der Anfangsinvestition, Eingriff in die Privatsphäre und Befürchtungen hinsichtlich des Einhaltens des Leistungsversprechens werden aus Nutzersicht angeführt. Aus Anbietersicht wird die fehlende Schnittstelle bestätigt und zusätzlich der allgemein hohe Aufwand (Rechtskonformität, Verwaltungsaufwand) sowie schlechte Erfahrungen mit frühen Sharing-Angeboten genannt.

Die Expertenaussagen in den Kategorien begünstigende Rahmenbedingungen und Umweltwirkungen weichen voneinander ab: Einig sind sich die Experten im Bereich der begünstigenden Rahmenbedingungen hinsichtlich der $\mathrm{CO}_{2}-$ Besteuerung, Prozesseinfachheit und einer Verbesserung der Infrastruktur. Unterschiedliche Sichtweisen werden innerhalb der Themenbereiche gesetzliche Anreize und Impulse erkenntlich. Im Bereich der Umweltwirkungen stimmen die Experten hinsichtlich positiver Umweltauswirkungen aufgrund von Einsparungen und einem höheren Anteil an erneuerbaren Energieerzeugungsanlagen weitgehend überein. Abweichende Einschätzungen treten 


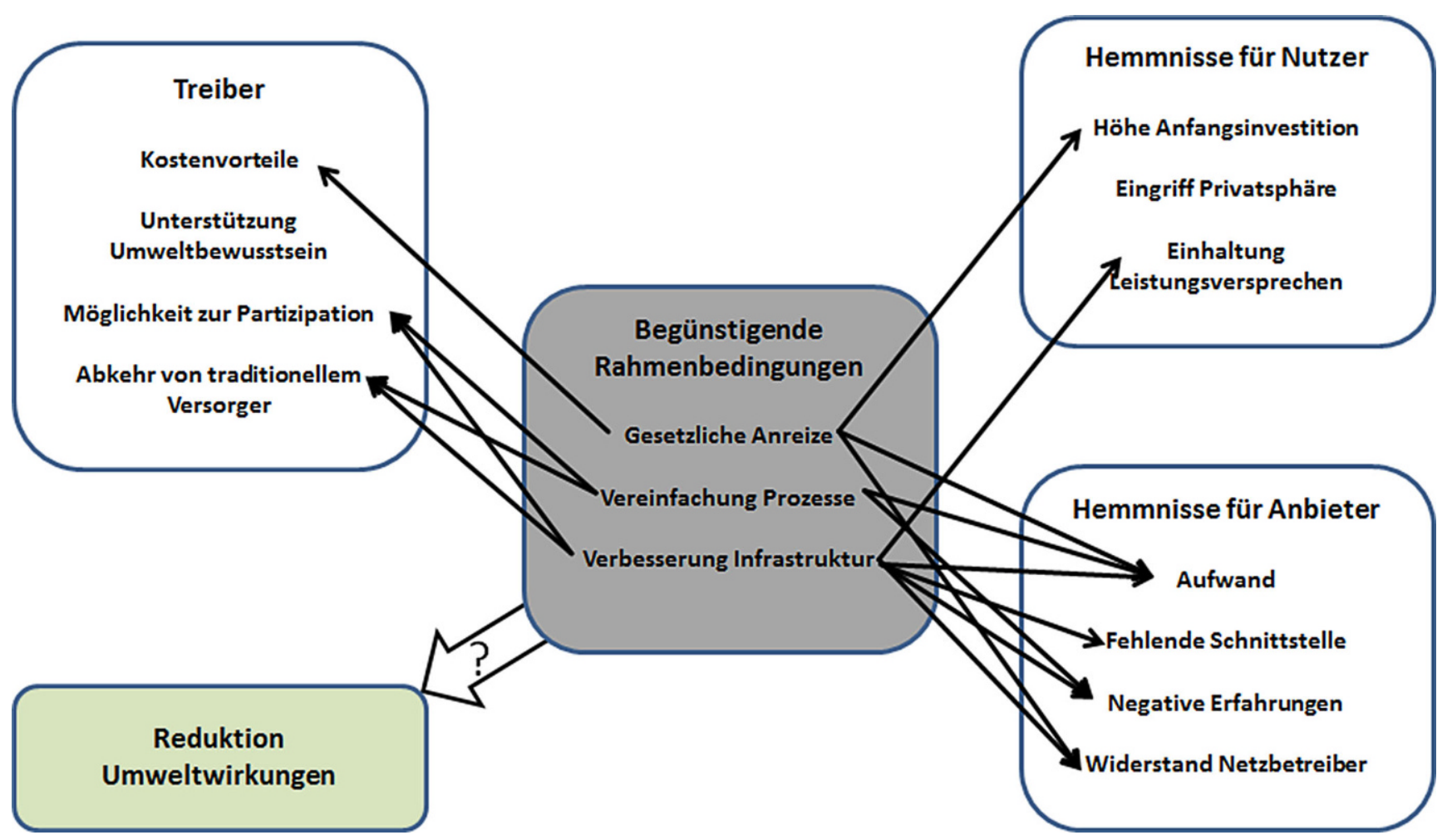

Abb. 1 Übersicht der Zusammenhänge zwischen den begünstigenden Rahmenbedingungen und den Treibern und Hemmnissen

hinsichtlich der Reboundeffekte auf. Insgesamt wirken die Aussagen im Bereich der Umweltwirkungen einseitig, da nahezu ausschließlich auf positive Auswirkungen der Sharing-Modelle eingegangen wird.

In Abb. 1 sind die Ergebnisse aus der Literaturanalyse und den Experteninterviews zusammengeführt. Das Verstärken von Treibern und das Abschwächen von Hemmnissen durch begünstigende Rahmenbedingungen ist durch Pfeile zwischen den einzelnen begünstigenden Rahmenbedingungen auf der einen Seite und den Treibern oder Hemmnissen auf der anderen Seite dargestellt. Die von den Experten formulierten Vorschläge zu gesetzlichen Anreizen wirken sich bspw. verstärkend auf den Treiber Kostenvorteile aus und schwächen die Hemmnisse Höhe der Anfangsinvestition, Aufwand und Widerstand beim Netzbetreiber. Vereinfachte Prozesse unterstützen die Treiber Abkehr vom traditionellen Versorger und die Möglichkeit zur Partizipation. Im Bereich der Hemmnisse wirkt sich dies auf der Anbieterseite je nach Ausgestaltung auf das Überwinden des erhöhten Aufwands und der negativen Erfahrungen mit ersten Sharing-Angeboten aus. Eine verbesserte Infrastruktur kann ebenfalls die Treiber Möglichkeit zur Partizipation und Abkehr vom traditionellen Versorger verstärken. Auf Anbieterseite werden dadurch sämtliche Hemmnisse reduziert und auf Nutzerseite die Befürchtung einer Nichteinhaltung des Leistungsversprechens verringert. Die begünstigenden Rahmenbedingungen führen insgesamt zu einem verstärkten Angebot bzw. Nutzung der Sharing-Angebote. Während die Experten davon ausgehen, dass die SharingAngebote grundsätzlich zu einer Reduzierung der Umweltwirkungen führen, wird dies in der Literatur kontrovers diskutiert und ist zu hinterfragen.

\subsection{Erkenntnisse der multiplen Fallanalyse}

Zur Auswertung und Gegenüberstellung werden die Fallbeispiele zuerst anhand der Kriterien zur Systematisierung der Sharing-Ansätze beschrieben, bevor die Ergebnisse zu Treibern, Hemmnissen und Umweltwirkungen im Folgenden präsentiert werden.

\subsubsection{Einordnung der Fallbeispiele}

Die drei Fallbeispiele repräsentieren die Varianten der P2PSharing-Ansätze in der Energiewirtschaft:

- Das Brooklyn Microgrid nutzt die Blockchain-Technologie zum Vernetzen der Teilnehmer in einem Microgrid in Brooklyn. Teilnehmer sind Nachfrager, die lokale Solarenergie erwerben und Prosumenten, die überschüssigen Strom aus solaren Energieerzeugungsanlagen anbieten. Die Prosumenten können wählen, ob sie überschüssigen Strom ausschließlich innerhalb des Mikrogrids oder auch an außenstehende Energieversorger verkaufen. Nachfra- 
ger können ihre Bezugsquellen auswählen und tägliche Budgets für den Einkauf lokaler Energie festlegen. Durch die Blockchain-Technologie erfolgen Abrechnung und Bezahlung ausschließlich zwischen dem Erzeuger und Nachfrager (Brooklyn Microgrid 2019a).

In den USA sind die gesetzlichen Voraussetzungen abhängig von den jeweiligen Bundesstaaten. Das Brooklyn Microgrid befindet sich in New York, wo seit dem Jahr 2019 durch das Clean Energy Communities Program kommunale Solar-Projekte in staatlich gefördert werden. Darüber ist Net-Metering für Projekte unter einer 750 Kilowatt-Kapazität möglich. Mithilfe des Net-Metering erhält der Betreiber einer Photovoltaikanlage eine 1:1 kW Gutschrift für überschüssigen Strom, der in das Netz eingespeist wird (Hess und Lee 2020).

- Powerpeers vernetzt als Online-Matching-Plattform dezentrale Energieerzeuger und Nachfrager in den Niederlanden. Zentrale Zielgruppe sind private Haushalte, die als Nachfrager und Erzeuger ein Netzwerk bilden. Nachfrager wählen aus den teilnehmenden Erzeugern aus und stellen sich ihren eigenen Strommix zusammen. Über eine App erfahren die Teilnehmer in Echtzeit, wieviel Strom sie von wem beziehen bzw. wieviel Strom sie an wen liefern. In Zeiten, in denen die Nachfrage das Angebot aus den teilnehmenden Anlagen übersteigt, wird Strom aus anderen erneuerbaren Quellen über Powerpeers bereitgestellt (Powerpeers 2020).

In den Niederlanden wird die Einspeisung aus erneuerbaren Energien durch garantierten Netzzugang und Gewährung eines Bonus gefördert (SDE + premium feed in scheme). Darüber hinaus werden erneuerbare Energien durch Darlehen und Steuervergünstigungen unterstützt. Kleine Anlagen werden zusätzlich durch die Möglichkeit des Net Metering begünstigt (Europäische Kommission 2020). Für Sharing Economy bestehen jedoch auch in den Niederlanden eine Reihe regulatorischer Herausforderungen. Butenko (2016) beschreibt in diesem $\mathrm{Zu}$ sammenhang u. a. die Erfordernis einer Versorgerlizenz für Prosumer und identifiziert eine regulatorische Lücke im Hinblick auf die Partizipation von Prosumern im niederländischen Elektrizitätsmarkt.

- Die SonnenCommunity ist eine Batteriespeicher-Community in Deutschland, deren Mitglieder Solaranlagen und Batteriespeicher besitzen. Teilnehmer speichern nicht verbrauchten Strom in ihren Batterien und speisen überschüssigen Strom bei Bedarf ins Netz ein. Eine zentrale Software ermittelt, aus welcher Batterie wann wieviel Strom ins Netz eingespeist wird. Zur Sicherstellung der Versorgung wird bei Bedarf Strom aus größeren erneuerbaren Energieanlagen (Windräder, Biogas-Anlagen und Solaranlagen) bezogen. Über die Eigenerzeugung hinausgehender Strombedarf wird bis zu einer vor- definierten Menge über eine Flatrate abgerechnet (sonnen GmbH 2020c).

Der Ausbau erneuerbarer Energien wird in Deutschland durch das Erneuerbare-Energien-Gesetz (EEG) gefördert. Allerdings läuft für viele Anlagen ab dem Jahr 2021 die EEG-Förderung aus und die EEG-Umlage ist auch für geteilten Strom zu leisten (Bundesregierung Deutschland 2020). In den Bundesländern gibt es darüber hinaus jeweils unterschiedliche Förderungen für erneuerbare Energieerzeugungsanlagen und Stromspeicher (siehe z.B. Solarwatt 2020). Die Förderungen sind allerdings an verschiedene Bedingungen geknüpft, die dazu dienen, dass der überschüssige Strom im deutschen Netz zur Verfügung steht. Bundesübergreifend stehen diesbezüglich günstige Kredite durch die KfW-Bank zur Verfügung.

Die Gegenüberstellung der Ausprägungen (siehe Tab. 5) zeigt Gemeinsamkeiten in der Kategorie „Geteiltes Gut“ der Sharing-Angebote: In allen Fallbeispielen werden die Kapazitäten erneuerbarer Erzeugungsanlagen geteilt, im Beispiel sonnenCommunity gibt es die Option, die Kapazität von Batteriespeichern allgemein zur Speicherung und auch für Redispatch-Maßnahmen zur Verfügung zu stellen. Ebenso handelt es sich bei allen Fallbeispielen um ein ergebnisbasiertes Produktservicesystem und Daten und Informationen betreffen in allen Fällen Stromverbrauch und Strombereitstellung im Zeitverlauf. Ein wesentlicher Unterschied besteht beim Kriterium Raum/Ressourcen/Technologie, da Nachfrager die Sharing-Angebote des Brooklyn Microgrid und der Powerpeers ohne eigene Erzeugungsanlagen nutzen können, während für die Teilnahme an der sonnenCommunity eine Solaranlage mit Batteriespeicher zwingend erforderlich ist. Im Gegensatz zu Powerpeers bieten SonnenCommunity und Brooklyn Microgrid Informationen zur Weiterbildung für ihre Kunden bzw. für alle Interessierten an. Prinzipiell sind alle Fallbeispiele erweiterbar, indem weitere Nachfrager, Erzeuger und/oder Prosumenten an den Sharing-Angeboten teilnehmen. Darüber hinaus sind im Fall der der sonnenCommunity die Batteriespeicher modular aufgebaut und in 2,5 kWh Schritten erweiterbar.

Hinsichtlich der Marktorientierung ist neben der Ausprägung Online-Plattform allen Fallbeispielen die Profitorientierung gemein. Die Netzwerkgröße national der Powerpeers ist inhaltlich unabhängig vom Sharing-Angebot. Demgegenüber ergeben sich die lokale Ausprägung aus dem dezentralen Charakter des Brooklyn Microgrid und die nationale Ausprägung der sonnenCommunity aus dem zugrundeliegenden Prinzip, lokale Verfügbarkeitsunterschiede auszunutzen.

Bezüglich der Kundensegmente in der Kategorie Marktstruktur weisen alle Fallbeispiele dieselbe Grundform auf: Die drei Plattformbetreiber bieten Privatkunden (B2C) die 
Tab. 5 Systematisierung der Fallbeispiele

\begin{tabular}{|c|c|c|c|}
\hline Projektname & Brooklyn Microgrid & Powerpeers & SonnenCommunity \\
\hline \multicolumn{4}{|c|}{ Allgemeine Informationen } \\
\hline Land & $\mathrm{USA}^{\mathrm{a}}$ & Niederlande ${ }^{\mathrm{d}}$ & Deutschland ${ }^{\mathrm{f}}$ \\
\hline Startzeitpunkt & $2016^{\mathrm{a}}$ & $2016^{\mathrm{d}}$ & $2015^{\mathrm{g}}$ \\
\hline $\begin{array}{l}\text { Ziel/ } \\
\text { Schlüsselaktivitäten }\end{array}$ & $\begin{array}{l}\text { P2P-Energiehandelsplatt-form zur Ver- } \\
\text { netzung dezentraler Solarstromanbieter } \\
\text { und -nachfrager unter Verwendung der } \\
\text { Blockchain-Technologie }\end{array}$ & $\begin{array}{l}\text { P2P-Energiehandelsplattform } \\
\text { zur Zuordnung von Erzeugern } \\
\text { zu Nachfragern }^{\mathrm{d}}\end{array}$ & $\begin{array}{l}\text { P2P-Energiehandelsplattform zur } \\
\text { gemeinschaftlichen Nutzung von } \\
\text { Solaranlagen und Batteriespeichern }\end{array}$ \\
\hline \multicolumn{4}{|l|}{ Geteiltes Gut } \\
\hline Material/Energie & $\begin{array}{l}\text { Materielle Güter: Kapazität erneuerbarer } \\
\text { Erzeugungsanlagen }^{\mathrm{a}}\end{array}$ & $\begin{array}{l}\text { Materielle Güter: Kapazität er- } \\
\text { neuerbarer Erzeugungsanlagen }{ }^{\mathrm{d}}\end{array}$ & $\begin{array}{l}\text { Materielle Güter: Kapazität von } \\
\text { Solaranlagen mit verbundenem } \\
\text { Stromspeicher }^{f}\end{array}$ \\
\hline Produktservicesystem & $\begin{array}{l}\text { Ergebnisbasiertes Produktservicesystem: } \\
\text { Inanspruchnahme Erzeugungsanlagen } \\
\text { für bestimmte Dauer, Erzeugungsanla- } \\
\text { gen im Besitz der Erzeuger }{ }^{\mathrm{a}}\end{array}$ & $\begin{array}{l}\text { Ergebnisbasiertes Produktser- } \\
\text { vicesystem: Inanspruchnahme } \\
\text { Erzeugungsanlagen für be- } \\
\text { stimmte Dauer, Erzeugungs- } \\
\text { anlagen im Besitz der Erzeuger }\end{array}$ & $\begin{array}{l}\text { Ergebnisbasiertes Produktservice- } \\
\text { system: Inanspruchnahme der Er- } \\
\text { zeugungsanlagen und Speicher für } \\
\text { bestimmte Dauer, Erzeugungsan- } \\
\text { lagen und Speicher im Besitz von } \\
\text { Prosumenten }\end{array}$ \\
\hline $\begin{array}{l}\text { Raum/Ressourcen/ } \\
\text { Technologie }\end{array}$ & $\begin{array}{l}\text { Erzeuger: Dachfläche für } \\
\text { Solaranlagen, Solaranlagen, Smart-Me- } \\
\text { ter, } \\
\text { Nachfrager: Smart-Meter, App mit Ver- } \\
\text { bindung zum Konto. } \\
\text { Alle: Internetzugang, Transactive Grid } \\
\text { Element zur Verbindung des Smart-Me- } \\
\text { ter mit lokalem Energiemarkt }^{\mathrm{c}}\end{array}$ & $\begin{array}{l}\text { Erzeuger: Smart-Meter, Anlagen } \\
\text { und hierfür benötigten Raum/ } \\
\text { Fläche } \\
\text { Nachfrager: Smart-Meter, App }{ }^{\mathrm{d}}\end{array}$ & $\begin{array}{l}\text { (Dach-) Fläche für } \\
\text { Erzeugungsanlagen, Erzeugungsanla- } \\
\text { gen, Speicher, Smart Meter }\end{array}$ \\
\hline $\begin{array}{l}\text { Daten und Informa- } \\
\text { tion }\end{array}$ & $\begin{array}{l}\text { Informationen zu Erzeugung und Ver- } \\
\text { brauch über } A p^{c}\end{array}$ & $\begin{array}{l}\text { Teilnehmen erhalten Visualisie- } \\
\text { rungen im Zeitverlauf, wessen } \\
\text { Strom sie bezogen bzw. an wen } \\
\text { sie geliefert haben }^{\mathrm{d}}\end{array}$ & $\begin{array}{l}\text { Informationen zu Verbrauch und } \\
\text { Erzeugung }{ }^{\mathrm{h}}\end{array}$ \\
\hline $\begin{array}{l}\text { Wissen/ } \\
\text { Weiterbildung }\end{array}$ & Kenntnis zu Verbrauch und Erzeugung ${ }^{\mathrm{c}}$ & - & $\begin{array}{l}\text { Informationen zur Energiewende, } \\
\text { erneuerbaren } \\
\text { Energien, Photovoltaik und } \\
\text { Stromspeichern }{ }^{\mathrm{i}}\end{array}$ \\
\hline Erweiterbarkeit & $\begin{array}{l}\text { Entwicklung Verbraucher zu Prosumen- } \\
\text { ten, Ergänzung von Anlagen }{ }^{\mathrm{b}}\end{array}$ & $\begin{array}{l}\text { Entwicklung Verbraucher zu } \\
\text { Prosumenten, Ergänzung von } \\
\text { Anlagen }^{\mathrm{d}}\end{array}$ & $\begin{array}{l}\text { Ergänzung von Anlagen, modularer } \\
\text { Aufbau der Batterie (erweiterbar in } \\
2,5 \mathrm{kWh} \mathrm{Schritten})^{\mathrm{j}}\end{array}$ \\
\hline Lebensdauer & Entspricht der Lebensdauer der Anlagen & $\begin{array}{l}\text { Entspricht der Lebensdauer der } \\
\text { Anlagen }\end{array}$ & $\begin{array}{l}\text { Entspricht der Lebensdauer der Anla- } \\
\text { gen }\end{array}$ \\
\hline \multicolumn{4}{|l|}{ Marktorientierung } \\
\hline Ertragsmodell & Profitorientiert $\mathrm{a}^{\mathrm{a}, \mathrm{c}}$ & Profitorientiert $^{\mathrm{d}}$ & Profitorientiert ${ }^{\mathrm{f}}$ \\
\hline Netzwerkgröße & Lokal $^{\mathrm{a}}$ & National $^{\mathrm{d}}$ & National ${ }^{\mathrm{f}}$ \\
\hline $\begin{array}{l}\text { Online/offline Platt- } \\
\text { form }\end{array}$ & Online Plattform ${ }^{\mathrm{b}}$ & Online Plattform ${ }^{\mathrm{d}}$ & Online-Plattform ${ }^{\mathrm{f}}$ \\
\hline \multicolumn{4}{|l|}{ Marktstruktur } \\
\hline Betreiber & $\begin{array}{l}\text { Blockchain-Technologie Exergy von } \\
\text { LO3 }^{\text {a }}\end{array}$ & Powerpeers ${ }^{\mathrm{d}}$ & Sonnen $\mathrm{GmbH}^{\mathrm{f}}$ \\
\hline Partner & Energieversorger Con Edison ${ }^{\mathrm{a}}$ & Vattenfall $^{\mathrm{e}}$ & $\begin{array}{l}\text { Verteilnetzbetreiber und } \\
\text { Energieversorger }^{\mathrm{g}}\end{array}$ \\
\hline Kundensegment & $\mathrm{B} 2 \mathrm{C} / \mathrm{C} 2 \mathrm{C} / \mathrm{C} 2 \mathrm{~B}^{\mathrm{a}}$ & $\mathrm{B} 2 \mathrm{C} / \mathrm{C} 2 \mathrm{C}^{\mathrm{d}}$ & $\mathrm{B} 2 \mathrm{C} / \mathrm{C} 2 \mathrm{C}^{\mathrm{f}}$ \\
\hline
\end{tabular}

a (Brooklyn Microgrid 2019a)

${ }^{\mathrm{b}}$ (LO3 energy 2018)

c (Brooklyn Microgrid 2019b)

d (Powerpeers 2020)

e (Vattenfall AB 2016)

f (sonnen GmbH 2020c)

$\mathrm{g}$ (sonnen GmbH 2020e)

h (sonnen GmbH 2020a)

i (sonnen GmbH 2020b)

j (sonnen GmbH 2020d) 
Möglichkeit, sich mit anderen Privatkunden (C2C) zu vernetzen. Zusätzlich wird im Brooklyn-Microgrid den Prosumenten die Möglichkeit gegeben, Strom an Energieversorger außerhalb des Netzwerks zu verkaufen (C2B).

\subsubsection{Treiber und Hemmnisse in den Fallbeispielen}

In diesem Abschnitt wird abgeglichen, inwieweit die aus der Literatur und den Experteninterviews gewonnenen Treiber und Hemmnisse in den Fallbeispielen relevant sind. Die analysierten Treiber und Hemmnisse lassen sich Tab. 6 und 7 entnehmen. Auf die Hemmnisse aus Anbietersicht (Aufwand, fehlende Schnittstelle, Erfahrungen, Widerstand Netzbetreiber) wird nicht weiter eingegangen, da diese im Zuge der Umsetzung überwunden wurden.

Eine Übersicht zum Vorhandensein der Treiber in den Fallbeispielen ist aus Tab. 6 ersichtlich. Diese Darstellung zeigt bereits, dass die erste Theorie in Bezug auf die Treiber bestätigt werden kann: Es existieren sowohl von der Ausgestaltung des Sharing-Angebots abhängige Treiber als auch Treiber, die sich in allen Sharing-Angeboten finden. Im Brooklyn Microgrid und bei Powerpeers können dieselben drei der vier Treiber identifiziert werden: Durch das Angebot aus dezentralen erneuerbaren Erzeugungsanlagen werden die Teilnehmer in ihrem Umweltbewusstsein bestätigt und die Abkehr von traditionellen Energieversorgern ermöglicht. Zudem besteht die Möglichkeit zur Partizipation unabhängig vom Besitz eigener Erzeugungsanlagen. Die sonnenCommunity bietet ebenfalls die Möglichkeit, sich vom traditionellen Energieversorger abzukehren und das Umweltbewusstsein der Teilnehmer durch die Nutzung erneuerbarer Energien zu unterstützen. Da eigene Erzeugungsanlagen und Speicher erforderlich sind, ist die Möglichkeit zur Partizipation ohne eigene Anlagen allerdings nicht gegeben. Inwieweit fallspezifische Kostenvorteile gegeben sind, lässt sich nicht abschließend bewerten. Sonnen stellt allerdings eine allgemeine Beispielrechnung auf der Website zu Verfügung. Bei den Sharing-Angeboten mit Eigenerzeugungsanlagen sind die spezifischen Voraussetzungen der Teilnehmer ausschlaggebend. Festhalten lässt sich, dass Powerpeers und Brooklyn Microgrid nicht auf Kostenvorteile bei der Darstellung ihres Angebots eingehen, während die sonnenCommunity anhand von Beispielrechnungen erläutert, dass sich der Speicher innerhalb weniger Jahre rentiere (sonnen GmbH 2020c). Darüber hinaus betrifft aufgrund der geografischen Lage ausschließlich Teilnehmer der sonnenCommunity das Argument zur Weiternutzung von Photovoltaikanlagen, die das Ende der EEGFörderung erreicht haben.

Einschränkend sei bereits an dieser Stelle angemerkt, dass das Umweltbewusstsein der Nutzer bereits durch das Angebot von erneuerbarem Strom und damit unabhängig vom Sharing-Gedanken unterstützt wird. Jedoch verbinden Nutzer Sharing-Angebote häufig mit Umweltvorteilen, weshalb eine Verstärkung des Effekts durch das Sharing-Angebot angenommen werden kann. Zum tatsächlichen Nutzen für die Umwelt wird auf die Ausführungen im Abschn. 4.2.3 Beeinflussung der Umweltwirkungen durch die Fallbeispiele verwiesen.

Tab. 7 zeigt die in den Fallbeispielen identifizierten Hemmnisse aus Nutzersicht und bestätigt ebenfalls die erste zu untersuchende Theorie: Es existieren einerseits Hemmnisse, die übergeordnet mit dem Sharing-Ansatz zusammenhängen, und andererseits Hemmnisse, die von der Ausgestaltung abhängig sind. Bei Powerpeers und Brooklyn Microgrid sind Anfangsinvestitionen möglich, aber nicht zwingend. Demgegenüber sind zur Teilnahme an der sonnenCommunity Investitionen in Solarmodule und Batteriespeicher erforderlich und gehen damit über die Anforderungen an Prosumenten in den anderen beiden Fallbeispielen hinaus. Für ihr Sharing-Angebot benötigen alle Plattformbetreiber in den Fallbeispielen Informationen zum aktuellen Stromverbrauch und zur Stromerzeugung ihrer Teilnehmer. Da Individuen Eingriffe in ihre Privatsphäre unterschiedlich empfinden, ist die Einstufung in Tab. 7 lediglich eine Abstufung des Ausmaßes. Durch den Einsatz der Blockchain-Technologie im Brooklyn Microgrid kann die Abrechnung und Bezahlung direkt zwischen den Teilnehmern stattfinden, sodass am wenigsten Informationen der Teilnehmer zentral vom Betreiber benötigt
Tab. 6 Treiber in den Fallbeispielen

Tab. 7 Hemmnisse aus Nutzersicht in den Fallbeispielen

\begin{tabular}{llll}
\hline & Brooklyn Microgrid & Powerpeers & SonnenCommunity \\
\hline Kostenvorteile & - & - & $(\mathrm{X})$ \\
Unterstützung Umweltbewusstsein & $\mathrm{X}$ & $\mathrm{X}$ & $\mathrm{X}$ \\
Möglichkeit zur Partizipation & $\mathrm{X}$ & $\mathrm{X}$ & - \\
Abkehr von traditionellem Versorger & $\mathrm{X}$ & $\mathrm{X}$ & $\mathrm{X}$ \\
\hline
\end{tabular}

\begin{tabular}{llll}
\hline & Brooklyn Microgrid & Powerpeers & SonnenCommunity \\
\hline Höhe der Anfangsinvestition & - & - & $\mathrm{X}$ \\
Eingriff Privatsphäre & - & $\mathrm{X}$ & $(\mathrm{X})$ \\
Einhaltung Leistungsversprechen & $(\mathrm{X})$ & $(\mathrm{X})$ & $(\mathrm{X})$ \\
\hline
\end{tabular}


Tab. 8 Übersicht Aspekte zu Verringerung der Umweltwirkungen in den Fallbeispielen

\begin{tabular}{llll}
\hline & Brooklyn Microgrid & Powerpeers & SonnenCommunity \\
\hline $\begin{array}{l}\text { Einsparungen, Konsumentenbildung, Verbrauchererziehung, reduzierter Res- } \\
\text { sourcenverbrauch }\end{array}$ & $\mathrm{X}$ & $\mathrm{X}$ & $(\mathrm{X})$ \\
Eigenverbrauchssteigerung, Maximierung des Verbrauchernutzens, Ressourcen & $(\mathrm{X})$ & $(\mathrm{X})$ & $(\mathrm{X})$ \\
Verhinderung Reboundeffekte & - & - & - \\
Verringerung des Produktionsdurchsatzes & - & - & - \\
Langlebigkeit, Haltbarkeit, Lieferantenauswahl nach Haltbarkeit & - & $(\mathrm{X})$ & $\mathrm{X}$ \\
Erweiterbarkeit & $\mathrm{X}$ & $\mathrm{X}$ \\
Verzicht auf nicht erneuerbare Ressourcen, Verringerung des Einsatzes nicht & & - \\
erneuerbarer Quellen & - & $\mathrm{X}$ \\
Reparaturfähigkeit & $\mathrm{X}$ & - \\
Keine Rabatte, kein Overselling & - & $\mathrm{X}$ \\
Wieder- und Weiterverwendbarkeit & $\mathrm{X}$ & - \\
Verringerung von Emissionen & - & $\mathrm{X}$ \\
Verringerung der Deponierung synthetischer Abfälle & & -
\end{tabular}

werden. Teilnehmer der sonnenCommunity übermitteln dem Plattformbetreiber zusätzlich zur Erzeugung und zur Nachfrage Informationen zum Speicherstand, geben jedoch untereinander keine Informationen preis. Demgegenüber tauschen die Teilnehmer von Powerpeers untereinander Informationen zum Stromverbrauch und zur Stromerzeugung aus und erleben somit den weitreichendsten Eingriff in die Privatsphäre. Das Hemmnis bezüglich des Einhaltens des Leistungsversprechens tritt vor allem bei neuen Marktakteuren auf. Verstärkt wird die Wahrnehmung dieses Hemmnisses durch Insolvenzrisiken, die sich bspw. in der Insolvenz des Anbieters des Heimspeichers Carteva Sonne bereits gezeigt haben (EUWID 2019). Es kann davon ausgegangen werden, dass dieses Hemmnis zu Beginn der drei Sharing-Angebote stärker ausgeprägt war, jedoch mit zunehmender Dauer und Bekanntheit der Angebote abgenommen hat.

Die von den Experten angeführten Änderungen der Rahmenbedingungen beziehen sich auf Deutschland, lassen sich jedoch verallgemeinernd für alle Fallbeispiele als begünstigend erachten. Bspw. unterstützen gesetzliche Anreize in Form einer $\mathrm{CO}_{2}$-Besteuerung den Treiber Wirtschaftlichkeit in allen Fallbeispielen, da Strom aus erneuerbaren Energieträgern hierdurch Kostenvorteile gegenüber Strom aus konventionellen Energieträgern erhält. Dies trifft ebenso auf die vereinfachte Inbetriebnahme von dezentralen erneuerbaren Erzeugungsanlagen zu, die in allen Fallbeispielen zur Deckung der Stromnachfrage eingesetzt werden. Darüber hinaus ist auch eine verbesserte Infrastruktur (Internet, bidirektionaler Stromfluss) begünstigend für die Fallbeispiele.

\subsubsection{Beeinflussung der Umweltwirkungen durch die Fallbeispiele}

Anhand der Beschreibungen der Fallbeispiele wird deren möglicher Beitrag zur Verringerung von Umweltwirkungen abgeleitet (siehe Tab. 8). Alle Beispiele beinhalten die Aspekte Konsumentenbildung durch eine Visualisierung der Stromnachfrage, bieten keine Rabatte und betreiben kein Overselling, verzichten auf nicht erneuerbare Energieträger und tragen dadurch zur Verringerung von Emissionen bei. Die Flatrate für den Reststrombedarf der sonnenCommunity kann sich im Sinne der Verbrauchererziehung allerdings negativ, d.h. in Form eines gesteigerten Verbrauchs, auswirken (vgl. Bauknecht et al. 2017). Grundsätzlich sind alle Systeme erweiterbar, jedoch wird nur bei der sonnenCommunity auf die Erweiterbarkeit einzelner Systemkomponenten explizit Bezug genommen. Alle Fallbeispiele zielen auf die Maximierung des Nutzens der Ressource Erneuerbarer Erzeugungsanlagen. Aufgrund der Funktionsweise der Strommärkte entsteht hierdurch allerdings nur dann ein Umweltnutzen, wenn das Angebot an Strom aus erneuerbaren Energieträgern im Vergleich zur insgesamt kontrahierten erneuerbaren Strommenge knapp ist und ein Impuls für den zusätzlichen Bau erneuerbarer Erzeugungskapazitäten resultiert. Durch den Batteriespeicher der sonnenCommunity-Teilnehmer wird der Eigenverbrauch der Ressource Erneuerbare Erzeugungskapazität gesteigert, allerdings ist der Batteriespeicher als weitere Ressource erforderlich. Bei begrenztem Gesamtkapital können hieraus geringere erneuerbare Erzeugungskapazitäten resultieren (vgl. Griese et al. 2016).

Die identifizierten Treiber und Hemmnisse für die Sharing-Ansätze wirken sich nur indirekt auf die Aspekte zur Verringerung der Umweltwirkungen in den Fallbeispielen aus. Treiber und Hemmnisse beeinflussen die Anzahl der 
Nutzer der Fallbeispiele, was bedeutet, dass bspw. die Wirkung der Treiber sich in Form einer höheren Anzahl an Nutzern oder Anbietern von Sharing-Modellen äußert. Dadurch werden die Ausprägungen der Merkmale im Bereich der Umweltwirkungen in ihrer Gesamtheit lediglich in der Höhe, nicht aber in der Art beeinflusst. Insofern kann die zweite Theorie zur Auswirkung der Treiber und Hemmnisse auf die Umweltwirkungen anhand der Untersuchungen weder widerlegt noch bestätigt werden.

\section{Diskussion}

Die identifizierten Treiber und Hemmnisse stammen aus einer umfassenden, systematischen Literaturrecherche und auf darauf basierenden Experteninterviews. Die Ergebnisse der Experteninterviews sind aufgrund der Anzahl der Experten nicht repräsentativ, jedoch zielen die Interviews vielmehr auf das Abdecken verschiedener Sichtweisen innerhalb der Energiewirtschaft ab. Die Experten werden durch ihr Umfeld beeinflusst und eine Unvoreingenommenheit gegenüber Sharing-Modellen kann nicht sichergestellt werden. Im Rahmen der Auswertung der Interviews zeigt sich, dass die Experten trotz einer eingangs gegebenen Definition auf ihr implizites Verständnis des Begriffs Sharing Economy zurückgreifen. Dies lässt sich auf den Umstand zurückführen, dass eine Vielzahl an Synonymen und abweichenden Definitionen für Sharing Economy existiert. Sharing Economy wird deshalb auch als ,fuzzy concept“ (Plewnia und Guenther 2018) bzw. das Konzept als „muddiness“ (Ritter und Schanz 2019) erachtet.

Um trotz dieser voneinander abweichenden Definitionen eine Vergleichsmöglichkeit zu schaffen, wurden Fallbeispiele dreier verschiedener Ausrichtungen innerhalb der Sharing Economy untersucht. Die Analyse der Fallbeispiele beruht auf den Informationen der offiziellen Websites der Betreiber. Es lässt sich damit nicht vermeiden, dass auf Informationen zurückgegriffen wird, die Sharing-Modelle möglicherweise einseitig und vorteilhaft darstellen. Diesen Unsicherheiten wird mit der gezielten Analyse der Fallbeispiele nach verschiedenen Kriterien und Merkmalen entgegengewirkt. Insgesamt zielt der Anspruch der Analysen dementsprechend nicht auf Vollständigkeit ab, sondern dient der Identifikation von Treibern und Hemmnissen sowie dem Aufzeigen von Gemeinsamkeiten und Unterschieden verschiedener P2P-Sharing-Ansätze in der Elektrizitätswirtschaft. Brooklyn Microgrid als Vertreter der Blockchain-Lösungen und Powerpeers als Vertreter der Online-Matching-Plattformen weisen aufgrund vergleichbarer Schlüsselaktivitäten dieselben Treiber auf. Ein Unterschied besteht im Hemmnis Eingriff in die Privatsphäre, da der Verzicht auf einen zentralen Datenbankbetreiber das wesentliche Merkmal der Blockchain-Technology ist.
Treiber und Hemmnisse der SonnenCommunity weichen demgegenüber deutlich $\mathrm{ab}$, was insbesondere im Zwang zur Investition in eigene Speicher begründet ist.

Insgesamt ist zu hinterfragen, inwieweit die gewählten Fallbeispiele und damit die Bewertung der Theorie hinsichtlich der Existenz individueller und übergeordneter Treiber wie Hemmnisse repräsentativ für die Gruppe der P2P-Sharing-Ansätze sind. Vereinfachte Analysen am Beispiel Vandebron (Vandebron Energie B.V. 2017) und Piclo Match (mittlerweile inaktiv) (Open Utility Ltd. 2018) bestätigen die Einschätzungen für die Online-Matching-Plattformen, für die Blockchain-Lösungen und Batteriespeicher-Communities bedarf es weiterer Untersuchungen.

Der jeweilige Stellenwert und damit verbunden die unterschiedlich starken Auswirkungen der identifizierten Treiber und Hemmnisse sowie mögliche kausale Zusammenhänge wurden in dieser Analyse nicht betrachtet und bedürfen weiterer Forschung. Aus diesem Grund sollten die Präferenzen der Nutzer einbezogen werden, die in diesem Beitrag ausschließlich durch die Literaturrecherche berücksichtigt wurden. Die Praxis zeigt, dass bspw. fehlende Kostenvorteile durch die Möglichkeit aufgewogen werden können, sich von traditionellen Versorgern abzukehren (Open Utility Ltd. 2018). Ein Widerspruch besteht zwischen dem Treiber Abkehr vom traditionellen Versorger und dem Hemmnis in Form der Befürchtung, dass Leistungsversprechen durch neue/kleine Marktakteure nicht eingehalten werden können. Hinsichtlich der Bedeutung des Treibers Abkehr vom Versorger bietet die Analyse der anstehenden Veränderung von Powerpeers zu einer Marke des traditionellen Versorgers Vattenfall (Vattenfall AB 2019) Möglichkeit für weitergehende Analysen.

Begünstigende Rahmenbedingungen, die zu einer Verbesserung von Umweltwirkungen beitragen, lassen sich aus den Interviews nicht ableiten, da die Experten weitgehend von positiven Umweltwirkungen der Sharing Economy ausgehen. Darüber hinaus beziehen sich die Experten und die anfängliche Literaturrecherche hinsichtlich der potentiellen Umweltwirkungen hauptsächlich auf die Umweltfolgen des $\mathrm{CO}_{2}$-Ausstoßes. Wie in den meisten Sharing-Ansätzen in der Energiewirtschaft ist die Nutzung erneuerbarer Erzeugungsanlagen Gegenstand aller drei Fallbeispiele. Die Verringerung der Emissionen ist in erster Linie nicht auf das eigentliche Sharing-Angebot, sondern auf die Nutzung erneuerbarer Energien zurückzuführen und führt nur dann zu übergeordneten Einsparungen, wenn durch das SharingAngebot zusätzliche erneuerbare Strommengen resultieren. Somit konnte die Theorie zur Auswirkung von Treibern und Hemmnissen auf die Umweltwirkungen weder bestätigt noch widerlegt werden und bedarf weiterer Untersuchungen.

Die identifizierten Treiber und Hemmnisse sind nur teilweise spezifisch für die Energiewirtschaft, teils treffen sie 
auf Sharing-Angebote bzw. sogar Geschäftsmodelle allgemein zu. Nichtsdestotrotz werden sie in der Literatur bzw. von den Experten als besonders relevant für Sharing-Angebote in der Energiewirtschaft erachtet. Dementsprechend können Sharing-Anbieter durch Analyse der möglichen Treiber und Hemmnisse die Erfolgsaussichten ihrer Angebote in der Energiewirtschaft hinterfragen. Die identifizierten Treiber können gezielt zur Werbung von Teilnehmern eingesetzt werden. Aus Nutzersicht bestehende Hemmnisse sollten, wenn möglich, von den Anbietern selbst vermieden werden, während zum Abbau der aus Anbietersicht bestehenden Hemmnisse begünstigende Rahmenbedingungen durch den Gesetzgeber geschaffen werden sollten. Das Hemmnis fehlender Plattformen haben bspw. ein Teil der Sharing-Anbieter als Geschäftsmodell erkannt, indem sie sich zusätzlich als Technologie-Anbieter etablieren wollen (z.B. Open Utility und Powerpeers).

Weitere Analysen zu Treibern und Hemmnissen bieten die Möglichkeit, Wissenschaft und Praxis im Bereich der Sharing Economy enger zu verknüpfen. Durch Ergänzungen der Systematisierungen der Geschäftsmodelle der Sharing Economy (z.B. allgemein auf Basis der Arbeiten von Ritter und Schanz (2019) und am Beispiel der Energiewirtschaft auf Basis der Arbeiten von Plewnia und Guenther (2018)) lassen sich konkrete Implikationen für die Ausgestaltung erfolgreicher Geschäftsmodelle in der Praxis ableiten. Insbesondere im Hinblick auf Treiber und Hemmnisse bezüglich der Umweltwirkungen von Sharing Economy Bedarf es zusätzlicher Analysen, da die vorliegende Arbeit lediglich eine indirekte Wirkung von Treibern und Hemmnissen identifiziert. Für Sharing-Angebote innerhalb und außerhalb der Energiewirtschaft gilt es die Umweltwirkungen der Sharing-Angebote in der Praxis zu erheben, um eine solide Basis für die Beschreibung von Treibern und Hemmnissen zum Ausschöpfen des (möglichen) Umweltnutzens zu ermöglichen.

\section{Schlussfolgerungen}

Die auf Basis der Literatur und der Experteninterviews gewonnenen Treiber und Hemmnisse für Sharing-Ansätze in der Energiewirtschaft finden sich in den Praxisbeispielen wieder. Während das Vorhandensein und die Bedeutung des Treibers Kostenvorteile in den Fallbeispielen hinterfragt werden müssen, lassen sich die Treiber Unterstützung des Umweltbewusstseins, Möglichkeit zur Partizipation und Abkehr von traditionellem Versorger in mindestens zwei der drei Fallbespiele identifizieren. Hinsichtlich der Identifikation der Hemmnisse aus Nutzersicht können nur zur Höhe der Anfangsinvestition konkrete Aussagen getroffen werden, da die Hemmnisse Eingriff in die Privatsphäre und Befürchtungen zur Einhaltung des Leistungsversprechens mit dem individuellen Empfinden der Nutzer verbunden sind. Hemmnisse aus Anbietersicht mussten im Rahmen der Umsetzung der Angebote bereits überwunden werden, weshalb keine Aussagen zu hohem Aufwand und fehlenden Schnittstellen getroffen werden können. Literatur und Experten fordern diesbezüglich vom Gesetzgeber, begünstigende Rahmenbedingungen zu schaffen.

Aussagen zu den Treibern und Hemmnissen bezüglich der Umweltwirkungen von Sharing-Angeboten bzw. zu den Umweltwirkungen selbst gestalten sich schwierig. Wie in der Literatur beschrieben, lassen sich auf Basis der allgemeinen Systematisierung von Sharing-Angeboten bzw. der Beschreibung von Fallbeispielen nur Erwartungen hinsichtlich der Umweltwirkungen formulieren, deren konkrete Auswirkungen in der Praxis bedürfen ausführlicher Analysen. Die im Rahmen der Analysen identifizierten Treiber und Hemmnisse wirken sich lediglich durch die Begünstigung einer größeren oder kleineren Teilnehmerzahl auf die Umweltwirkungen der Fallbeispiele aus, beeinflussen die Umweltwirkung selbst aber nicht.

Die Literaturarbeit, die Auswertung der Experteninterviews und die Analyse der Fallbeispiele offenbaren die Herausforderungen bei der Analyse von Ansätzen der Sharing Economy. Das Verständnis von Sharing Economy als Obergriff und nicht als Definition führt zu einer großen Heterogenität in Wissenschaft und Praxis, was die Ableitung allgemeingültiger und spezifischer Treiber und Hemmnisse erschwert. Die zunehmenden Systematisierungsansätze schaffen die erforderliche Grundlage für die differenzierte inhaltliche Auseinandersetzung und sollten um Treiber und Hemmnisse ergänzt werden. Auf dieser Basis lassen sich Handlungsempfehlungen für erfolgreiche Sharing-Ansätze in der Energiewirtschaft erarbeiten. Dennoch bieten bereits die vorliegenden Analysen erste Hinweise für die Praxis, wie Sharing-Ansätze in der Elektrizitätswirtschaft ausgestaltet sein müssen, um die Aussichten für eine erfolgreiche Umsetzung zu erhöhen. Um durch Sharing-Ansätze die Transformation der Energiesysteme voranzutreiben, bedarf es darüber hinaus seitens des Gesetzgebers einen Wandel durch die Schaffung begünstigender Rahmenbedingungen für sowohl die Anbieter- als auch Nutzerseite. Monetäre Vorteile und bürokratische Vereinfachungen sowie mehr Transparenz in der Kostengestaltung können für eine größere Teilnahmebereitschaft auf Nutzerseite beitragen. Für die Politik bedeutet das, für mehr finanzielle Anreize und Transparenz zu sorgen. Im gleichen Zug sollten für die Anbieterseite die Verringerung des Verwaltungsaufwands und Verbesserungen in der Infrastruktur (Internet und lokale Speicher) forciert und dabei klare Richtlinien für die Rechtskonformität neuer Vertragsmodelle zur Verfügung gestellt werden. 
Funding Open Access funding enabled and organized by Projekt DEAL.

Interessenkonflikt P. Szichta und I. Tietze geben an, dass kein Interessenkonflikt besteht.

Open Access Dieser Artikel wird unter der Creative Commons Namensnennung 4.0 International Lizenz veröffentlicht, welche die Nutzung, Vervielfältigung, Bearbeitung, Verbreitung und Wiedergabe in jeglichem Medium und Format erlaubt, sofern Sie den/die ursprünglichen Autor(en) und die Quelle ordnungsgemäß nennen, einen Link zur Creative Commons Lizenz beifügen und angeben, ob Änderungen vorgenommen wurden.

Die in diesem Artikel enthaltenen Bilder und sonstiges Drittmaterial unterliegen ebenfalls der genannten Creative Commons Lizenz, sofern sich aus der Abbildungslegende nichts anderes ergibt. Sofern das betreffende Material nicht unter der genannten Creative Commons Lizenz steht und die betreffende Handlung nicht nach gesetzlichen Vorschriften erlaubt ist, ist für die oben aufgeführten Weiterverwendungen des Materials die Einwilligung des jeweiligen Rechteinhabers einzuholen.

Weitere Details zur Lizenz entnehmen Sie bitte der Lizenzinformation auf http://creativecommons.org/licenses/by/4.0/deed.de.

\section{Literatur}

Bauknecht D, Bürger V, Ritter D, Vogel M, Langniß O, Brenner T, Chvanova E, Geier L (2017) Bestandsaufnahme und orientierende Bewertung dezentraler Energiemanagementsysteme. Climate Change, Dessau

Bocken NMP, Short SW, Rana P, Evans S (2014) A lit erature and practice review to develop sustainable business model archetypes. J Clean Prod 42-56. https://doi.org/10.1016/j.jclepro.2013.11.039

Bogner A, Littig B, Menz W (2014) Interviews mit Experten: Eine praxisorientierte Einführung. Springer VS, Wiesbaden

Booth A (2006) "Brimful of STARLITE": toward standards for reporting literature searches. J Med Libr Assoc 94:421-430

Brinkmann C, Schulz S (2011) Die Energiegenossenschaften: Ein kooperatives Beteiligungsmodell. Bochum. https://www.energiege nossenschaften-gruenden.de/fileadmin/user_upload/downloads/ Artikel_und_Materialien/Die_Energiegenossenschaften._Ein_ kooperatives_Beteiligungsmodell_01.pdf

Bundesregierung Deutschland (2020) Gesetz für den Ausbau erneuerbarer Energien: Erneuerbare-Energien-Gesetz - EEG 2017

Butenko A (2016) Sharing energy. Eur j risk regul 7:701-716. https:// doi.org/10.1017/S1867299X00010138

Celik B, Roche R, Bouquain D, Miraoui A (2018) Decentralized neighborhood energy management with coordinated smart home energy sharing. IEEE Trans Smart Grid 9:6387-6397. https://doi.org/ 10.1109/TSG.2017.2710358

Daunorienė A, Drakšaitė A, Snieška V, Valodkienė G (2015) Evaluating sustainability of sharing economy business models. Procedia Soc Behav Sci 213:836-841. https://doi.org/10.1016/j.sbspro. 2015.11.486

Eisenhardt KM, Graebner ME (2007) Theory building from cases: opportunities and challenges. AMJ 50:25-32. https://doi.org/10. 5465/amj.2007.24160888

Europäische Kommission (2020) Renewable energy policy database and support. www.res-legal.eu/home/. Zugegriffen: 14. Juli 2020

EUWID (2019) Nachfolgegesellschaft von Caterva meldet Insolvenz an. https://www.euwid-energie.de/nachfolgegesellschaft-voncaterva-meldet-insolvenz-an/. Zugegriffen: 17. Juli 2020

Gährs S, Knoefel J (2020) Stakeholder demands and regulatory framework for community energy storage with a focus on Germany. Energy Policy 144:111678. https://doi.org/10.1016/j.enpol.2020. 111678
Gossen M, Pentzien J, Peuckert J (2019) What use is it really for sustainability?: Potentials and impacts of peer-to-peer sharing in the domains of accommodation and mobility. NachhaltigkeitsManagementForum 27:125-138. https://doi.org/10.1007/s00550019-00488-8

Griese K-M, Wawer T, Böcher R (2016) Suffizienzorientierte Geschäftsmodelle am Beispiel von Stromspeichern: Zukunftsfähige Ansätze in der Energiewirtschaft. Z Energiewirtsch 40:66-67

Hackbarth A, Löbbe S (2020) Attitudes, preferences, and intentions of German households concerning participation in peer-to-peer electricity trading. Energy Policy 138:111238. https://doi.org/10. 1016/j.enpol.2020.111238

Hamari J, Sjöklint M, Ukkonen A (2016) The sharing economy: why people participate in collaborative consumption. J Assn Inf Sci Tec 67:2047-2059. https://doi.org/10.1002/asi.23552

Han X, Sun L, Tao Y, Zhao J, Wang G, Yuan D (2020) Distributed energy-sharing strategy for Peer-to-Peer microgrid system. J Energy 146:4020033. https://doi.org/10.1061/(ASCE)EY.1943-7897. 0000665

Heinrich-Böll-Stiftung, Green European Foundation, European Renewable Energy Federation, le Monde diplomatique (2018) Energieatlas 2018. Heinrich-Böll-Stiftung, Berlin

Helfferich C (2019) Leitfaden- und Experteninterviews. In: Baur N, Blasius J (Hrsg) Handbuch Methoden der empirischen Sozialforschung. Springer, Wiesbaden, S 669-686

Hess DJ, Lee D (2020) Energy decentralization in California and New York: conflicts in the politics of shared solar and community choice. Renew Sustain Energy Rev 121:109716. https://doi.org/ 10.1016/j.rser.2020.109716

Hoffmann TM (2016) Motivation im Führungskontext von Sozialunternehmen. Internationalisierung und Management. Springer, Wiesbaden

Hoffmann E, Mohaupt F, Ortmanns M (2018) Akzeptanz von Speicherdienstleistungen und weiteren Energiedienstleistungen. Stand der Forschung aus sozialwissenschaftlicher Perspektive. Projekt ESQUIRE, Berlin

Kloppenburg S, Boekelo M (2019) Digital platforms and the future of energy provisioning: promises and perils for the next phase of the energy transition. Energy Res Soc Sci 49:68-73. https://doi.org/ 10.1016/j.erss.2018.10.016

Kröhling A (2017) Digitalisierung - Technik für eine nachhaltige Gesellschaft? In: Hildebrandt A, Landhäußer W (Hrsg) CSR und Digitalisierung: Der digitale Wandel als Chance und Herausforderung für Wirtschaft und Gesellschaft. Springer Gabler, Berlin, Heidelberg, S 23-49

Li B, Wan C, Yuan K, Song Y (2019) Demand response for integrating distributed energy resources in transactive energy system. Energy Procedia 158:6645-6651. https://doi.org/10.1016/j.egypro.2019. 01.040

LO3 energy (2018) Exergy business whitepaper. LO3 energy, Brooklyn

Long C, Wu J, Zhou Y, Jenkins N (2018) Aggregated battery control for peer-to-peer energy sharing in a community Microgrid with PV battery systems. Energy Procedia 145:522-527. https://doi. org/10.1016/j.egypro.2018.04.076

Mayring P, Brunner E (2009) Qualitative Inhaltsanalyse. In: Buber R, Holzmüller HH (Hrsg) Qualitative Marktforschung: Konzepte - Methoden - Analysen, Bd. 2. Gabler GWV, Wiesbaden, S 669-680

Microgrid B (2019a) BMG 101 - Brooklyn Microgrid 101. https:// www.brooklyn.energy/bmg-101. Zugegriffen: 24. März 2020

Microgrid B (2019b) FAQs - Brooklyn Microgrid. https://www. brooklyn.energy/faqs. Zugegriffen: 24. März 2020

Nicklaß T, Schmack U, Teucher T (2017) Flexibilität als neue Währung: Die Verbindung von Wärme-, Strom- und Mobilitätsnetz am Beispiel der Digital Energy Solutions (DES). In: Hildebrandt A, Landhäußer W (Hrsg) CSR und Digitalisierung: Der digitale Wandel als Chance und Herausforderung für Wirtschaft und Gesellschaft. Springer Gabler, Berlin, Heidelberg, S 227-243 
Ohlhorst D (2018) Akteursvielfalt und Bürgerbeteiligung im Kontext der Energiewende in Deutschland: Das EEG und seine Reform. In: Holstenkamp L, Radtke J (Hrsg) Handbuch Energiewende und Partizipation. Springer, Wiesbaden, S 101-124

Open Utility Ltd (2018) Local grid charging-exploring the incentivisation of local energy. Open Utility Ltd, London

Otuoze AO, Mustafa MW, Larik RM (2018) Smart grids security challenges: Classification by sources of threats. J Electr Syst Inf Technol 5:468-483. https://doi.org/10.1016/j.jesit.2018.01.001

Parra D, Norman SA, Walker GS, Gillott M (2017) Optimum community energy storage for renewable energy and demand load management. Appl Energy 200:358-369. https://doi.org/10.1016/j. apenergy.2017.05.048

Pasimeni F (2019) Community-based adoption and diffusion of microgrids: analysis of the Italian case with agent-based model. JASSS. https://doi.org/10.18564/jasss.3950

Peer CD, Engel D, Wicker SB (2014) Hierarchical key management for multi-resolution load data representation. In: IEEE International Conference on Smart Grid Communications (SmartGridComm) Venice, 3-6 Nov. 2014 IEEE, Piscataway, S 926-932

Pires Klein L, Krivoglazova A, Matos L, Landeck J, de Azevedo M (2020) A novel Peer-to-Peer energy sharing business model for the Portuguese energy market. Energies 13:125. https://doi.org/ $10.3390 /$ en 13010125

Plewnia F (2019) The energy system and the sharing economy: interfaces and overlaps and what to learn from them. Energies 12:339. https://doi.org/10.3390/en12030339

Plewnia F, Guenther E (2018) Mapping the sharing economy for sustainability research. Manag Decis 56:570-583. https://doi.org/10. 1108/MD-11-2016-0766

Poppen S (2015) Energiegenossenschaften und deren Mitglieder: Erste Ergebnisse einer empirischen Untersuchung. Münster. http://hdl. handle.net/10419/123711

Powerpeers (2020) About powerpeers. https://www.powerpeers.nl/ about. Zugegriffen: 30. März 2020

Ritter M, Schanz H (2019) The sharing economy: a comprehensive business model framework. J Clean Prod 213:320-331. https:// doi.org/10.1016/j.jclepro.2018.12.154

Solarwatt (2020) Förderung für Photovoltaik Stromspeicher nach Bundesland. https://www.solarwatt.de/stromspeicher/foerderung $\mathrm{Zu}-$ gegriffen: 09. Juli 2020

Sonnen GmbH (2020a) Häufig gestellte Fragen. https://sonnen.de/ haeufig-gestellte-fragen/. Zugegriffen: 25. März 2020

Sonnen GmbH (2020b) Regenerative Energien verstehen und nutzen. https://sonnen.de/wissen/. Zugegriffen: 25. März 2020

Sonnen GmbH (2020c) SonnenCommnunity - die größte StromSharing Plattform. https://sonnen.de/sonnencommunity/. Zugegriffen: 25. März 2020

Sonnen GmbH (2020d) Stromspeicher von sonnen. https://sonnen.de/ stromspeicher/. Zugegriffen: 25. März 2020
Sonnen GmbH (2020e) Vom Stromspeicher zum Energieversorger. https://sonnen.de/ueber-uns/unternehmen/. Zugegriffen: 25. März 2020

Sun SI, Kiaee M, Norman S, Wills R (2018) Self-sufficiency ratio: an insufficient metric for domestic PV-battery systems? Energy Procedia 151:150-157

Sundararajan A (2016) The sharing economy: the end of employment and the rise of crowd-based capitalism. MIT Press, Cambridge

Tang Y, Zhang Q, Li H, Li Y, Liu B (2019) Economic analysis on repurposed EV batteries in a distributed PV system under sharing business models. Energy Procedia 158:4304-4310. https://doi.org/10. 1016/j.egypro.2019.01.793

Tietze I (2020) Sharing economy in the German energy transition. Int J Bus Glob 24:392-412

Tikka V, Mashlakov A, Kulmala A, Repo S, Aro M, Keski-Koukkari A, Honkapuro S, Järventausta P, Partanen J (2019) Integrated business platform of distributed energy resources-Case Finland. Energy Procedia 158:6637-6644. https://doi.org/10.1016/j. egypro.2019.01.041

Untersteller F (2016) CSR und Energiewirtschaft aus baden-württembergischer Perspektive. In: Hildebrandt A, Landhäußer W (Hrsg) CSR und Energiewirtschaft. Springer, Berlin Heidelberg, S 1-16

Vandebron Energie BV (2017) Vandebron is een duurzaam energiebedrijf gevestigd in Amsterdam. https://vandebron.nl/about. Zugegriffen: 8. Dez. 2017

Vattenfall AB (2016) Vattenfall launches energy sharing marketplace powerpeers. https://group.vattenfall.com/press-and-media/ news--press-releases/newsroom/2016/vattenfall-launches-energysharing-marketplace-powerpeers. Zugegriffen: 30. März 2020

Vattenfall AB (2019) Vattenfall will unite another energy supplier brand under one name. https://group.vattenfall.com/press-andmedia/news--press-releases/newsroom/2019/vattenfall-will-uniteanother-energy-supplier-brand-under-one-name. Zugegriffen: 31. März 2020

Yin RK (2018) Case study research: design and methods. SAGE, Los Angeles, London, New Delhi, Singapore, Washington, DC

Zhang C, Wu J, Long C, Cheng M (2017) Review of existing Peerto-Peer energy trading projects. Energy Procedia 105:2563-2568. https://doi.org/10.1016/j.egypro.2017.03.737

Zhou Y, Wu J, Long C (2018) Evaluation of peer-to-peer energy sharing mechanisms based on a multiagent simulation framework. Appl Energy 222:993-1022. https://doi.org/10.1016/j.apenergy. 2018.02.089

Zhou Y, Wu J, Long C, Ming W (2020) State-of-the-art analysis and perspectives for Peer-to-Peer energy trading. Engineering. https:// doi.org/10.1016/j.eng.2020.06.002 